\title{
Impact of improved energy resolution on DUNE sensitivity to neutrino non-standard interactions
}

\author{
Sabya Sachi Chatterjee, ${ }^{a}$ P.S. Bhupal Dev ${ }^{b}$ and Pedro A.N. Machado ${ }^{c}$ \\ ${ }^{a}$ Institute for Particle Physics Phenomenology, Department of Physics, Durham University, \\ Durham, DH1 3LE, U.K. \\ ${ }^{b}$ Department of Physics and McDonnell Center for the Space Sciences, Washington University, \\ St. Louis, MO 63130, U.S.A. \\ ${ }^{c}$ Theoretical Physics Department, Fermi National Accelerator Laboratory, \\ Batavia, IL 60510, U.S.A. \\ E-mail: sabya.s.chatterjee@durham.ac.uk, bdev@wustl.edu, \\ pmachado@fnal.gov
}

ABStract: The full physics potential of the next-generation Deep Underground Neutrino Experiment (DUNE) is still being explored. In particular, there have been some recent studies on the possibility of improving DUNE's neutrino energy reconstruction. The main motivation is that a better determination of the neutrino energy in an event-by-event basis will translate into an improved measurement of the Dirac $C P$ phase and other neutrino oscillation parameters. To further motivate studies and improvements on the neutrino energy reconstruction, we evaluate the impact of energy resolution at DUNE on an illustrative new physics scenario, viz. non-standard interactions (NSI) of neutrinos with matter. We show that a better energy resolution in comparison to the ones given in the DUNE conceptual and technical design reports may significantly enhance the experimental sensitivity to NSI, particularly when degeneracies are present. While a better reconstruction of the first oscillation peak helps disentangling standard $C P$ effects from those coming from NSIs, we find that the second oscillation peak also plays a nontrivial role in improving DUNE's sensitivity.

Keywords: Beyond Standard Model, Neutrino Physics

ArXiv EPrint: 2106.04597 


\section{Contents}

1 Introduction 1

2 Theoretical framework 3

$\begin{array}{lll}3 & \text { Experimental setup } & 6\end{array}$

$\begin{array}{lll}4 & \text { Simulation details } & 7\end{array}$

$\begin{array}{llr}5 & \text { Improved energy resolution } & 7\end{array}$

6 Numerical results $\quad 10$

$\begin{array}{lll}7 & \text { Discussion } & 15\end{array}$

8 Conclusions $\quad 18$

\section{Introduction}

Decades of solar, atmospheric, accelerator and reactor neutrino experiments have firmly established the phenomenon of neutrino oscillations among the three flavors [1]. After the recent discovery of the relatively large reactor mixing angle $\theta_{13}$, the three-neutrino oscillation paradigm has entered a new precision era, where the known oscillation parameters are being measured with an ever-increasing accuracy. At the same time, several short, medium and long-baseline neutrino oscillation experiments, either running or in the pipeline, are poised to resolve the sub-dominant effects in oscillation data sensitive to the currently unknown oscillation parameters, namely the Dirac $C P$ phase $\delta_{\mathrm{CP}}$, the sign of the atmospheric neutrino mass-squared difference $\Delta m_{32}^{2}$, and the octant of the atmospheric mixing angle $\theta_{23}$.

Unraveling the neutrino properties within the three-neutrino framework has been a great success so far, when analyzing either a single experiment or the entirety of current neutrino oscillation data in terms of global fits [2-4]. Nevertheless, the quest for new physics beyond the Standard Model (SM) in the neutrino sector remains remarkably vibrant. Indeed, the phenomenon of neutrino oscillations itself suggests nonzero neutrino masses, which requires some beyond the SM (BSM) physics. Therefore, exploiting the full potential of the current and next-generation neutrino experiments to probe BSM physics is an important ongoing research topic.

One interesting model-independent framework for parameterizing BSM physics in the neutrino sector is the so-called non-standard interactions (NSI) [5]; for reviews on various aspects of NSI, see refs. [6-9]. The NSI framework is an effective field theory below the 
electroweak scale that may encode the oscillation phenomenology of an entire class of new physics scenarios; see e.g. refs. [10-16] for specific ultraviolet (UV)-complete models of NSI. The presence of NSI in either neutrino production, detection or propagation through matter can crucially affect the interpretation of the experimental data $[17,18]$. At the very least, it could serve as a foil for the three-neutrino oscillation scheme [19-34]. Therefore, a better understanding of the NSI effects is essential for the success of the future precision neutrino experiments in answering the open questions in neutrino physics.

Since neutral-current interactions affect neutrino propagation coherently, long-baseline neutrino experiments with a well-understood beam and trajectory are an ideal place to probe matter NSI effects. Numerous studies have been performed to this effect exploring the NSI prospects in the long-baseline experiments; see e.g. refs. [35-59] and references therein. In fact, a mismatch in the determination of the $\delta_{\mathrm{CP}}$ extracted from the latest data of the two running long-baseline neutrino experiments, $\mathrm{NO} \nu \mathrm{A}$ [60] and $\mathrm{T} 2 \mathrm{~K}$ [61], might already be hinting towards some possible indications of nonzero NSI [56, 57].

The next-generation long-baseline experiment DUNE [62, 63], with its high-intensity neutrino beam, huge statistics, wide-band spectrum and improved systematic uncertainties, is in an excellent position to probe matter NSI [44, 45, 50, 53] and BSM physics involving neutrinos in general [64]. At present, the full physics potential of liquid argon time projection chamber (LArTPC) detectors is still being explored. Several developments and novel proposals have been put forward recently to bolster the capabilities of these detectors, such as sub-MeV ionization energy detection in ArgoNeuT [65] and other LArTPC detectors [66], sub-GeV study of atmospheric neutrinos [67], and novel $\nu_{\tau}$ detection strategies [68-70] at DUNE. These studies are aimed at further enhancing the DUNE sensitivity to BSM physics.

Of particular interest for the present paper is the possibility of having an improved reconstruction of the neutrino energy by collecting all ionization energy and/or identifying each particle in an event-by-event basis [71, 72]. These studies show that the current energy resolution as reported in the original DUNE Conceptual Design Report (CDR) [62] and the DUNE Technical Design Report (TDR) [73] can be considerably improved by up to a factor of 2 to 3 . It has also been shown that the better energy resolution will contribute to a more precise determination of the Dirac $C P$ phase [71].

In this work, we will show for the first time that not only the determination of the standard oscillation parameters will benefit from an improved energy resolution, but also DUNE's sensitivity to new physics. We will take NSI as a general example of new physics and will analyze how the experimental sensitivity changes when going from the CDR and TDR resolutions to an improved neutrino energy resolution scenario. For the improved case, we will take the best energy reconstruction results of ref. [72], which leverages particle identification and the detection of all ionization energy, including de-excitation gammas from argon nuclei. Our findings show that the better neutrino energy resolution significantly boosts the DUNE sensitivity to NSI, improving its constraints by up to $25-30 \%$ for several NSI parameters. The improved energy resolution leads to a better reconstruction of both first and second oscillation peaks. While the first peak comprises most of the statistical power in DUNE, we find that the second peak provides a non-negligible contribution 
to the sensitivity, helping better disentangle standard $C P$ effects from those coming from NSIs. ${ }^{1}$ These findings hold true for single NSI, with or without associated $C P$ phases, and also when multiple NSI parameters are considered at the same time.

The rest of the paper is organized as follows: in section 2, we briefly review the theoretical framework of NSIs. In section 3, we review the DUNE experimental setup. In section 4 , we give our simulation details. In section 5, we discuss the improved energy resolution. Our main numerical results are presented in section 6 . In section 7 , we present a qualitative discussion of our results. Our conclusions are given in section 8 .

\section{Theoretical framework}

Non-standard interactions, or NSIs, first recognized in ref. [5], is an effective field theory below the weak scale, without explicit $\mathrm{SU}(2)_{L}$ invariance, that encompasses four-fermion operators involving at least one neutrino field. Logically, we can divide NSIs into two categories: charged-current $(\mathrm{CC})$ operators, in which the leptonic current has a neutrino and a charged lepton; and neutral-current (NC) NSI where the leptonic current has two neutrino fields. While CC-NSIs may affect neutrino production and detection [76], NCNSIs induce non-trivial matter effects and modify the dynamics of flavor conversion in matter [5]. Long-baseline experiments like DUNE are strongly affected by matter effects, and thus are expected to have exceptional sensitivity to NC-NSIs.

While the ultraviolet completion of NC-NSIs can arise either via heavy mediators (see e.g. refs. $[13,16,77])$ or light mediators $[10,11,14,78]$, we adopt an agnostic approach here and analyze the low energy phenomenology irrespective of model building considerations. To be more precise, NC-NSI can be described by dimension-six operators as,

$$
\mathcal{L}_{\mathrm{NC}-\mathrm{NSI}}=-2 \sqrt{2} G_{F} \varepsilon_{\alpha \beta}^{f C}\left(\bar{\nu}_{\alpha} \gamma^{\mu} P_{L} \nu_{\beta}\right)\left(\bar{f} \gamma_{\mu} P_{C} f\right),
$$

where $\alpha, \beta=e, \mu, \tau$ denote the neutrino flavors, $f=e, u, d$ indicate the matter fermions, $C=L, R$ corresponds to the chirality of the fermionic $f$ - $f$ current, and $\varepsilon_{\alpha \beta}^{f C}$ are the strengths of the NSI. The hermiticity of the Lagrangian requires

$$
\varepsilon_{\beta \alpha}^{f C}=\left(\varepsilon_{\alpha \beta}^{f C}\right)^{*} .
$$

It is worth mentioning that the diagonal $(\alpha=\beta)$ NSIs are always real whereas the nondiagonal $(\alpha \neq \beta)$ NSIs are in general complex as they always appear along with their associated $C P$-phases.

For neutrino propagation in the Earth, the potential induced by matter is the sum of the potentials induced by electrons, protons and neutrons. Since only the vector part of the current is relevant for coherent forward scattering, it is convenient to define

$$
\varepsilon_{\alpha \beta} \equiv \sum_{f=e, u, d} \varepsilon_{\alpha \beta}^{f} \frac{N_{f}}{N_{e}} \equiv \sum_{f=e, u, d}\left(\varepsilon_{\alpha \beta}^{f L}+\varepsilon_{\alpha \beta}^{f R}\right) \frac{N_{f}}{N_{e}},
$$

\footnotetext{
${ }^{1}$ For discussions related to the relevance of DUNE's second oscillation peak, see e.g. refs. [71, 74] in the context of standard oscillation physics and ref. [75] in the context of NSI.
} 
where $N_{f}$ is the number density of fermion $f$. For the crust of the Earth, which is what long baseline neutrinos travel through, we can assume neutral and isoscalar matter, implying $N_{n}=N_{p}=N_{e}$, in which case $N_{u}=N_{d}=3 N_{e}$. Therefore,

$$
\varepsilon_{\alpha \beta} \simeq \varepsilon_{\alpha \beta}^{e}+3 \varepsilon_{\alpha \beta}^{u}+3 \varepsilon_{\alpha \beta}^{d} .
$$

The presence of NSI modifies the effective Hamiltonian of neutrino propagation in matter, which in the flavor basis becomes

$$
H=U\left[\begin{array}{ccc}
0 & 0 & 0 \\
0 & k_{21} & 0 \\
0 & 0 & k_{31}
\end{array}\right] U^{\dagger}+V_{\mathrm{CC}}\left[\begin{array}{ccc}
1+\varepsilon_{e e} & \varepsilon_{e \mu} & \varepsilon_{e \tau} \\
\varepsilon_{e \mu}^{*} & \varepsilon_{\mu \mu} & \varepsilon_{\mu \tau} \\
\varepsilon_{e \tau}^{*} & \varepsilon_{\mu \tau}^{*} & \varepsilon_{\tau \tau}
\end{array}\right]
$$

where $U$ is the PMNS matrix, which, in its standard parameterization, depends on three mixing angles $\left(\theta_{12}, \theta_{13}\right.$, and, $\left.\theta_{23}\right)$ and one $C P$-phase $\left(\delta_{\mathrm{CP}}\right)$. The quantities $k_{21} \equiv \Delta m_{21}^{2} / 2 E$ and $k_{31} \equiv \Delta m_{31}^{2} / 2 E$ represent the solar and atmospheric wavenumbers, where $\Delta m_{i j}^{2} \equiv$ $m_{i}^{2}-m_{j}^{2}$ and $E$ is the neutrino energy. $V_{\mathrm{CC}}$ is the CC matter potential,

$$
V_{\mathrm{CC}}=\sqrt{2} G_{F} N_{e} \simeq 7.6 Y_{e} \times 10^{-14}\left[\frac{\rho}{\mathrm{g} / \mathrm{cm}^{3}}\right] \mathrm{eV},
$$

where $Y_{e}=N_{e} /\left(N_{p}+N_{n}\right) \simeq 0.5$ is the relative electron number density in Earth's crust and $\rho$ is the Earth matter density, which for DUNE we take as $\rho=2.848 \mathrm{~g} / \mathrm{cm}^{3}$.

For convenience we introduce the dimensionless quantity $\hat{v}=V_{\mathrm{CC}} / k_{31}$, which gauges the sensitivity to matter effects. Its absolute value

$$
|\hat{v}|=\left|\frac{V_{\mathrm{CC}}}{k_{31}}\right| \simeq 8.8 \times 10^{-2}\left[\frac{E}{\mathrm{GeV}}\right],
$$

appears in the analytical expressions of the $\nu_{\mu} \rightarrow \nu_{e}$ appearance probability and $\nu_{\mu} \rightarrow \nu_{\mu}$ survival probability, as we will see below.

Let us now discuss the appearance and survival probabilities relevant for the longbaseline experiment DUNE. In the presence of NSI, one can realize that the mixing angle $\theta_{13}$, the parameter $\hat{v}$ at DUNE and non-diagonal NSIs $\left|\varepsilon_{\alpha \beta}\right|$ are small. While the first two have similar size $\eta \sim 0.1$, where $\eta$ is simply a small expansion parameter, for neutrino energies of a couple of $\mathrm{GeV}$ or so, the latter cannot be much larger than that. Therefore we can perform an expansion on those parameters considering them to be roughly of the same magnitude $\mathcal{O}(\eta)$. We can also define $\alpha \equiv \Delta m_{21}^{2} / \Delta m_{31}^{2}= \pm 0.03$, which would then be $\mathcal{O}\left(\eta^{2}\right)$. Note that at DUNE $\hat{v}$ can be relatively large, particularly for the high energy tail of the spectrum, and thus the expansions that we will perform on small $\hat{v}$ may break down if $\left|\varepsilon_{\alpha \beta}\right|$ is not much smaller than 1. Nevertheless it is still useful to do the expansion in order to understand the role of energy resolution in the search for NSIs. Our numerical analysis is, however, exact and does not rely on any such expansions.

Let us discuss the appearance and disappearance oscillation probabilities up to the third order in $\eta$ given in refs. [21, 53, 79]. We will write the NSI parameters in general as $\varepsilon_{\alpha \beta}=\left|\varepsilon_{\alpha \beta}\right| e^{i \phi_{\alpha \beta}}$ for non-diagonal NSIs $(\alpha \neq \beta)$ while for the diagonal NSI couplings 
we simply have $\varepsilon_{\alpha \alpha}$ as a real parameter (its sign is physical). First, we start with the appearance probability,

$$
\begin{aligned}
P\left(\nu_{\mu} \rightarrow \nu_{e}\right) \simeq & \sin ^{2} 2 \theta_{13} s_{23}^{2} f^{2}+2 \alpha s_{13} \sin 2 \theta_{12} \sin 2 \theta_{23} f g \cos \left(\Delta+\delta_{\mathrm{CP}}\right) \\
& +8 \hat{v} s_{13} s_{23}\left\{\left|\varepsilon_{e \mu}\right|\left[s_{23}^{2} f^{2} \cos \left(\phi_{e \mu}+\delta_{\mathrm{CP}}\right)+c_{23}^{2} f g \cos \left(\Delta+\delta_{\mathrm{CP}}+\phi_{e \mu}\right)\right]\right. \\
& \left.\quad+\left|\varepsilon_{e \tau}\right| s_{23} c_{23}\left[f^{2} \cos \left(\phi_{e \tau}+\delta_{\mathrm{CP}}\right)-f g \cos \left(\Delta+\delta_{\mathrm{CP}}+\phi_{e \tau}\right)\right]\right\},
\end{aligned}
$$

where $\Delta \equiv \Delta m_{31}^{2} L / 4 E$ is the atmospheric oscillating frequency, $L$ being the baseline. For compactness, we have used the notation $s_{i j} \equiv \sin \theta_{i j}, c_{i j} \equiv \cos \theta_{i j}$, and,

$$
f \equiv \frac{\sin \left\{\left[\left(1-\hat{v}\left(1+\varepsilon_{e e}\right)\right] \Delta\right\}\right.}{1-\hat{v}\left(1+\varepsilon_{e e}\right)}, \quad g \equiv \frac{\sin \left[\hat{v}\left(1+\varepsilon_{e e}\right) \Delta\right]}{\hat{v}\left(1+\varepsilon_{e e}\right)} .
$$

The first line of eq. (2.8) is the standard approximated formula if $\varepsilon_{e e} \rightarrow 0$, see e.g. refs. [80-82], while the second and third lines are the modifications induced by NSIs. Notice that for small oscillation phase $\Delta$, both $f$ and $g$ are linear in $\Delta$. The leading term in $\left|\varepsilon_{e \tau}\right|$ tends to cancel for small $\Delta$, and thus we expect this NSI parameter to not strongly affect the high energy part of DUNE's far-detector spectrum. Therefore, the impact of $\left|\varepsilon_{e \tau}\right|$ may be relatively more pronounced in the second oscillation maximum. The observability of the second oscillation maximum is directly related to the precision of DUNE's energy reconstruction. We will see later that an improved energy resolution will significantly enhance the sensitivity to $\left|\varepsilon_{e \tau}\right|$, and that both first and second oscillation peaks contribute appreciably for this improvement. This effect is not present for $\left|\varepsilon_{e \mu}\right|$, and we will confirm that with our numerical analysis.

The $\nu_{\mu} \rightarrow \nu_{\mu}$ disappearance oscillation probability is affected by a different set of NSI parameters:

$$
\begin{aligned}
P\left(\nu_{\mu} \rightarrow \nu_{\mu}\right) \simeq 1 & -\sin ^{2} 2 \theta_{23} \sin ^{2} \Delta+\alpha c_{12}^{2} \sin ^{2} 2 \theta_{23} \Delta \sin 2 \Delta-4 s_{23}^{4} s_{13}^{2} \frac{\sin ^{2}[(1-\hat{v}) \Delta]}{(1-\hat{v})^{2}} \\
& -\frac{\sin ^{2} 2 \theta_{23} s_{13}^{2}}{(1-\hat{v})^{2}}\{\hat{v} \Delta \sin 2 \Delta+\sin [(1-\hat{v}) \Delta] \sin [(1+\hat{v}) \Delta]\} \\
- & 2 \hat{v}\left|\varepsilon_{\mu \tau}\right| \cos \phi_{\mu \tau}\left(\sin ^{3} 2 \theta_{23} \Delta \sin 2 \Delta+2 \sin 2 \theta_{23} \cos ^{2} 2 \theta_{23} \sin ^{2} \Delta\right) \\
+ & {\left[\hat{v} \sin ^{2} 2 \theta_{23} \cos 2 \theta_{23}\left(\varepsilon_{\mu \mu}-\varepsilon_{\tau \tau}\right)-\frac{\hat{v}^{2}}{2} \sin ^{4} 2 \theta_{23}\left(\varepsilon_{\mu \mu}-\varepsilon_{\tau \tau}\right)^{2}\right] } \\
& \times\left(\Delta \sin 2 \Delta-2 \sin ^{2} \Delta\right) .
\end{aligned}
$$

The first two lines describe standard oscillations, while the effect of NSIs is encoded in the last 2 lines. The leading term on $\varepsilon_{\mu \tau}$ is also multiplied by $\cos \phi_{\mu \tau}$, see the third line in the equation above. Thus, if one marginalizes over the phase when presenting the sensitivity to this NSI parameter, the result will typically be weak for DUNE as the leading 
term can always be put to zero. In experiments where matter effects are more relevant like IceCube, $\hat{v} \gtrsim 1$, the expansion performed here breaks down and the impact of the $\phi_{\mu \tau}$ phase becomes less pronounced, see e.g. ref. [83]. It is also worth mentioning that the second term in the parenthesis is proportional to $\cos ^{2} 2 \theta_{23}$, which may be fairly suppressed as $\theta_{23}$ approaches maximality.

The leading $\varepsilon_{\mu \mu}-\varepsilon_{\tau \tau}$ term is suppressed by $\cos ^{2} 2 \theta_{23}$, and thus sensitivity to this parameter should present a strong dependence on $\theta_{23}$. Moreover, both leading and nextto-leading terms cancel out for small $\Delta$, and so we anticipate an important role in the experimental sensitivity to this parameter to be played by the second oscillation and the energy resolution as a consequence. Our numerical simulations will confirm this expectation as well. Also, note that the first term in parenthesis in the last line of eq. (2.10) is linear in $\varepsilon_{\mu \mu}-\varepsilon_{\tau \tau}$, whereas the second term is quadratic. This is the reason for the difference in the DUNE sensitivities to $\varepsilon_{\mu \mu}$ and $\varepsilon_{\tau \tau}$, when each NSI is considered at a time, as we will see later.

In the expressions above, for normal mass ordering, the sign of $\Delta, \alpha$ and $\hat{v}$ are all positive, while for inverted ordering they are all negative. Analogous expressions to those in eqs. (2.8) and (2.10) for $P\left(\nu_{\mu} \rightarrow \nu_{e}\right)$ and $P\left(\nu_{\mu} \rightarrow \nu_{\mu}\right)$ can be obtained for antineutrinos by flipping the sign of all $C P$ phases and $\hat{v}$. For concreteness, we will present all our numerical results for normal ordering (NO) only.

\section{Experimental setup}

DUNE is a future long-baseline accelerator neutrino experiment, where neutrinos are expected to travel a distance of $1300 \mathrm{~km}$ from the source at Fermilab to the far-detector placed deep underground at the Sanford Lab in South Dakota. In order to estimate the sensitivity of the experiment to NSIs, we adopt three benchmark experimental configurations for DUNE: the Conceptual Design Report configuration (CDR) [62, 84]; the more recent Technical Design Report configuration (TDR) [73, 85]; and a third which is the TDR configuration with improved energy resolution based on the findings of ref. [72], as we will discuss later, which we refer to as the Best Reconstruction (Best Rec.).

To perform the experimental simulations we have used the GLoBES package [86, 87] together with the NSI tool from ref. [88]. In all configurations we have assumed a 40 kton LArTPC detector. The CDR beam configuration assumes an $80 \mathrm{GeV}$ proton beam with $1.07 \mathrm{MW}$ beam power resulting in $1.47 \times 10^{21} \mathrm{POT} /$ year, while the TDR uses a $120 \mathrm{GeV}$ proton beam with 1.2 MW beam power resulting in $1.1 \times 10^{21} \mathrm{POT} /$ year. We have also assumed equal time of 3.5 years running in each forward and reverse horn configurations (that is, equal neutrino and antineutrino runtime), which results in 300 and 336 ktonMW-year exposures for the CDR and TDR setups, respectively. Incidentally, the TDR staged 7-year running gives the same 336 kton-MW-year exposure [73]. For the CDR and TDR simulations, specific details on systematic errors and efficiencies can be found in refs. $[84,85]$. 


\section{Simulation details}

In order to quantify the statistical sensitivity of all the numerical simulations performed in this work, we use the built-in $\chi^{2}$ function in GLoBES, which incorporates systematic uncertainties via pull parameters and penalty terms (see ref. [87] for details). The total $\chi^{2}$ is the sum of all four channels that can be studied at DUNE: muon disappearance and electron appearance, in both forward and reverse horn polarity (i.e. neutrino and antineutrino modes). We marginalize over SM and new physics parameters whenever specified to obtain the minimum $\Delta \chi^{2}$.

In all our numerical simulations we have used a line-averaged constant Earth matter density $\rho=2.848 \mathrm{gm} / \mathrm{cm}^{3}$ for DUNE following the PREM $[89,90]$ profile. Unless otherwise stated, we have used the following benchmark values of the standard three-neutrino oscillation parameters: $\Delta m_{21}^{2}=7.5 \times 10^{-5} \mathrm{eV}^{2}, \Delta m_{31}^{2}=2.51 \times 10^{-3} \mathrm{eV}^{2}, \sin ^{2} \theta_{12}=0.310$, $\sin ^{2} \theta_{13}=0.022$, and $\sin ^{2} \theta_{23}=0.56$. Moreover, throughout this paper for simplicity we have assumed normal mass ordering (NO). Note that our benchmark parameters closely agree with the current global fit results [2-4]. As the solar mixing parameters have very small impact on DUNE, we have fixed $\theta_{12}$ and $\Delta m_{21}^{2}$ to their above mentioned values. Atmospheric parameters $\Delta m_{31}^{2}, \theta_{23}$ and $\delta_{\mathrm{CP}}$ are always allowed to vary freely as their measurements are among the main goals of DUNE. We adopt a $3.7 \%$ uncertainty on the reactor angle, which is better constrained at experiments like Daya Bay [91] and RENO [92]. Finally, we assume a $5 \%$ uncertainty on the average matter density, slightly larger than the $2 \%$ uncertainty assumed in the TDR. Whenever appropriate, we also marginalize over some new physics parameters.

\section{Improved energy resolution}

Charged particles traversing LArTPC ionize the liquid argon, leading to free electrons which drift against the electric field and are collected by wires. The charge deposit is used to reconstruct objects, such as tracks or showers, which are then used for particle identification. As the amount of energy deposited depends on the particle momentum and mass, the identification, together with the overall energy deposited in the detector via ionization is used to reconstruct the energy of each individual particle. With those energies and momenta, the incoming neutrino energy is inferred. This is to say that the reconstruction of the incoming neutrino energy, a crucial step to understand neutrino oscillations, is not a simple business.

Recently, there has been some re-evaluation of the best resolution that could be achieved by the DUNE experiment [71, 72]. In particular, ref. [72] studies the best case scenario, regarding incoming neutrino energy reconstruction, if the detector is able to identify all particles in an event and if the small but frequent energy deposits from recoiled neutrons are also accounted for. The authors find that a significant enhancement, up to a factor of 4 , in the reconstruction with respect to the CDR values could be achieved. ${ }^{2}$

\footnotetext{
${ }^{2}$ Note that in ref. [72], besides the "best reconstruction," another possibility is also studied for reconstructing the neutrino energy, that is, adding all calorimetric energy of the hadronic system without relying on individual particle identification. It is observed that this method leads to a neutrino energy resolution comparable to the one in DUNE's TDR, as so we do not present this "Charge" case separately here.
} 

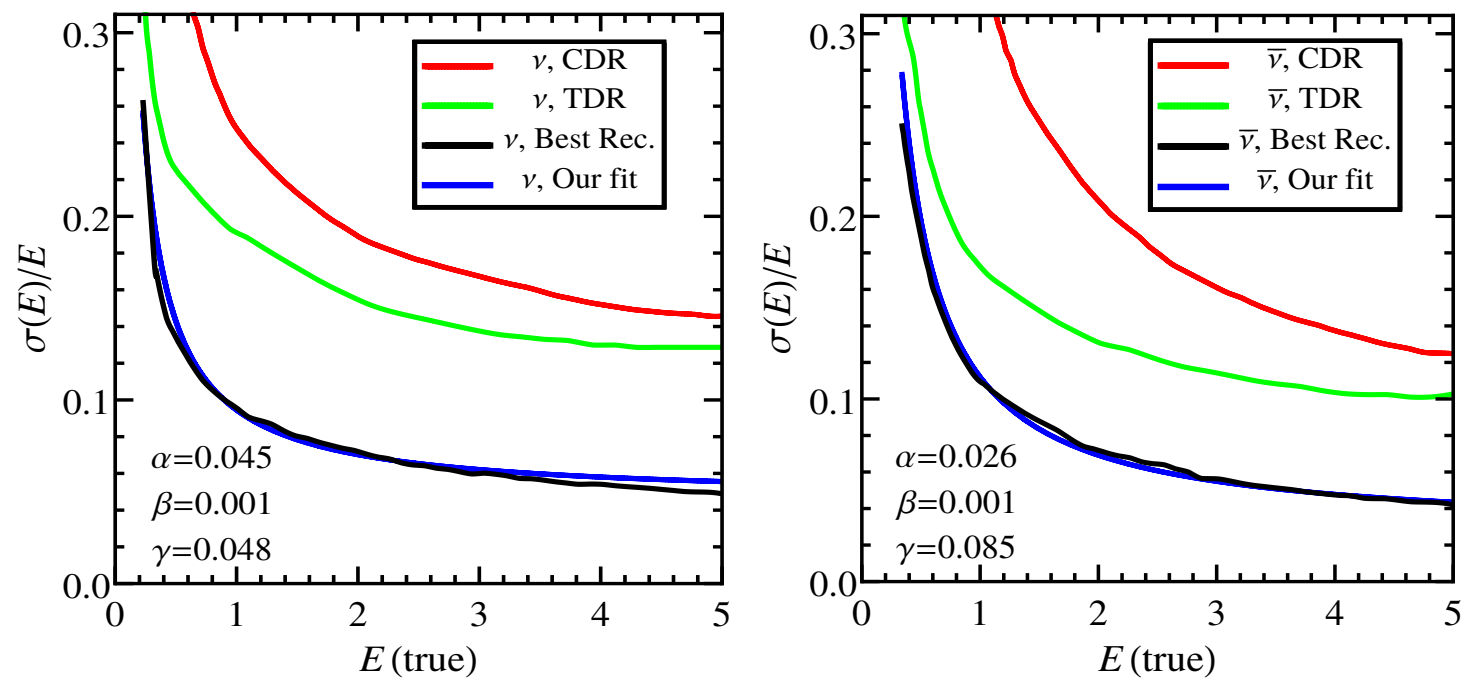

Figure 1. Energy resolution as a function of true neutrino energy. Left (right) panel corresponds to neutrinos (antineutrinos). The red and green curves depict the energy resolutions from DUNE's CDR and TDR, respectively, while the best reconstruction scenario from ref. [72] is shown in black. Our fit to the best reconstruction scenario is given by the blue curve. The numbers $\alpha, \beta$, and $\gamma$ in each panel correspond to the best fit values used in eq. (5.1).

In this paper, we are interested in understanding how much an improved energy resolution would enhance DUNE's sensitivity to new physics, particularly NSIs. In figure 1 we present the energy resolution for neutrinos (left panel) and antineutrinos (right panel) as a function of the true neutrino energy for the CDR (red line, worst resolution), and for the TDR (green line). We also show the best case scenario for the energy resolution obtained in ref. [72] (black line, labeled "Best Rec.") as well as a simple parameterization we perform to reproduce the best case results (blue line, labeled "Our fit"). Note that from figure 2 onwards we use our parameterization of the energy resolution in the Best Reconstruction case.

Our parameterization of the Best Reconstruction case is obtained by using an energy resolution function $R\left(E, E_{r}\right)=e^{-\left(E-E_{r}\right)^{2} / 2 \sigma^{2}} / \sigma \sqrt{2 \pi}$, where $E$ is the true neutrino energy, $E_{r}$ is the reconstructed energy, and the energy resolution $\sigma$ is given by

$$
\sigma(E) / \mathrm{GeV}=\alpha \cdot(E / \mathrm{GeV})+\beta \cdot \sqrt{E / \mathrm{GeV}}+\gamma,
$$

where, $(\alpha, \beta, \gamma)$ are the parameters for the fit: $(0.045,0.001,0.048)$ for neutrinos and $(0.026,0.001,0.085)$ for antineutrinos (cf. figure 1). We assume the same energy resolution for the appearance and disappearance modes. To simulate the neutral current, $\nu_{e}$ contamination, misidentified muon, and $\nu_{\mu} \rightarrow \nu_{\tau}$ backgrounds we have used the same energy resolution migration matrices as provided in refs. [84] (CDR) and [85] (TDR and Best Reconstruction).

As we can see from figure 1, the energy resolution is the worst for the CDR configuration, somewhat better for the TDR and improves considerably in the Best Reconstruction 

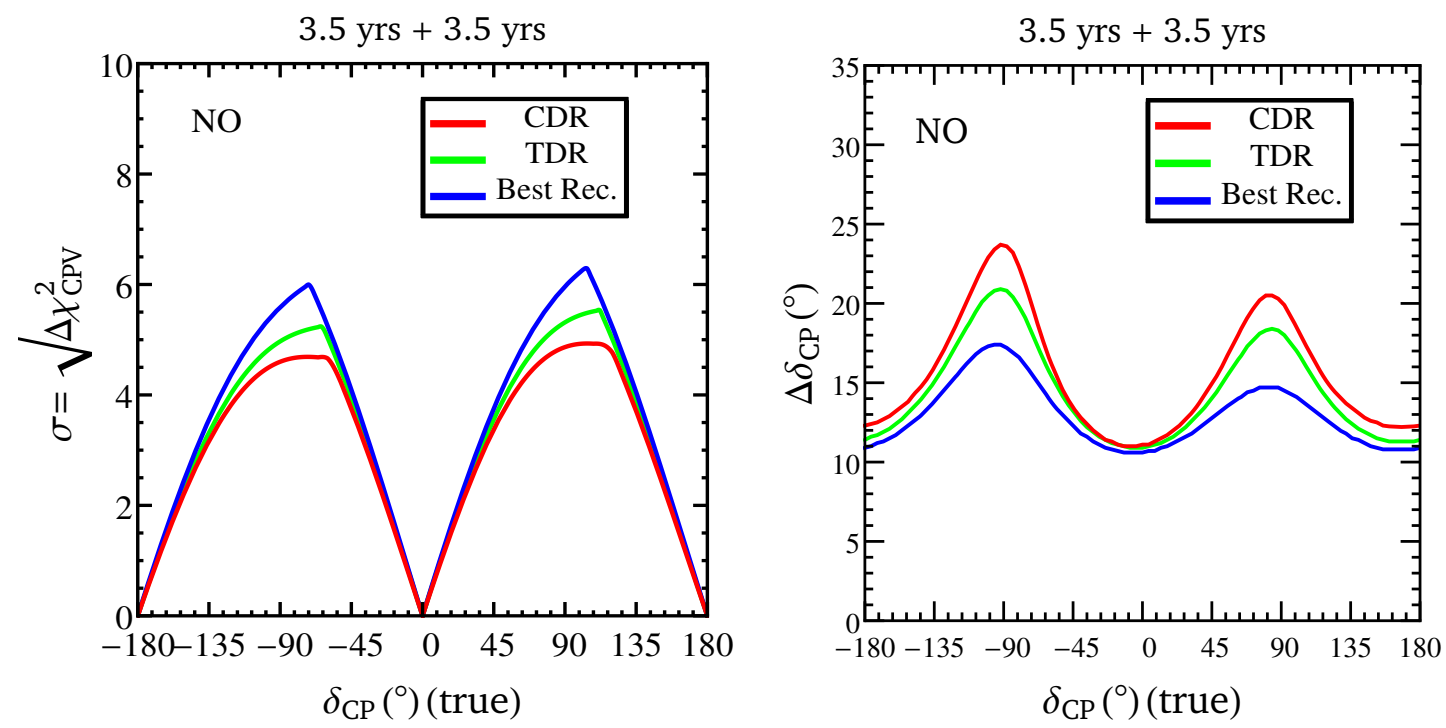

Figure 2. Left panel: $C P$ violation discovery $\left(\delta_{\mathrm{CP}}\right.$ (test) $\left.\neq 0, \pm \pi\right)$ potential of DUNE with different assumptions on the DUNE energy resolution. Right Panel: $1 \sigma\left(\Delta \chi^{2}=1\right)$ uncertainties on the true value of $\delta_{\mathrm{CP}}$ determined by DUNE. Red, green and blue curves represent the CDR, TDR and Best Reconstruction setup respectively. We have assumed normal ordering (NO) in the analysis for both plots.

case. Quantitatively, for neutrinos at $1 \mathrm{GeV}$ we have about 25\%,19\% and $9 \%$ energy resolution, respectively. For reference, near $0.9 \mathrm{GeV}$, an energy resolution of about $20 \%$ would completely wash out the second oscillation maximum.

A concrete example illustrating the importance of the energy resolution for DUNE is presented in figure, 2 , where we show, as a function of the true $\delta_{\mathrm{CP}}$ phase, the $C P$ violation sensitivity (left panel $)^{3}$ and the precision on $\delta_{\mathrm{CP}}$ achievable at DUNE at $1 \sigma\left(\Delta \chi^{2}=1\right.$, right panel) for 3.5 years in each neutrino and antineutrino mode. For both panels, the red, the green, and the blue lines correspond to the CDR, TDR, and the Best Reconstruction benchmarks. As discussed above we marginalize over all relevant oscillation parameters except the solar ones, as well as over the matter density. As we can see, the effect of a better energy resolution is non-negligible, substantially enhancing the $C P$ precision, in particular for maximal $C P$ violation. Quantitatively, for $\delta_{\mathrm{CP}}($ true $)=-90^{\circ}$, the $C P$ precision is $\sim 24^{\circ}$ for the CDR, $\sim 21^{\circ}$ for the TDR, and $\sim 17^{\circ}$ for the Best Reconstruction case. Similar findings have been reported previously in ref. [71].

It is important to mention that the $C P$ precision obtained here with DUNE's GLoBES simulation files [85] does not match the official DUNE version of the plot given in ref. [73]. The main reason seems to be the treatment of systematics related to the flux and/or cross section shape uncertainties. Compared to a full-fledged Monte Carlo simulation, the GLoBES treatment of spectral uncertainties in ref. [85] is somewhat simplified.

\footnotetext{
${ }^{3}$ The $C P$ violation sensitivity is defined as the statistical significance at which one can reject the test hypothesis of no $C P$ violation i.e., $\delta_{\mathrm{CP}}($ test $)=0$, or $\pm \pi$.
} 

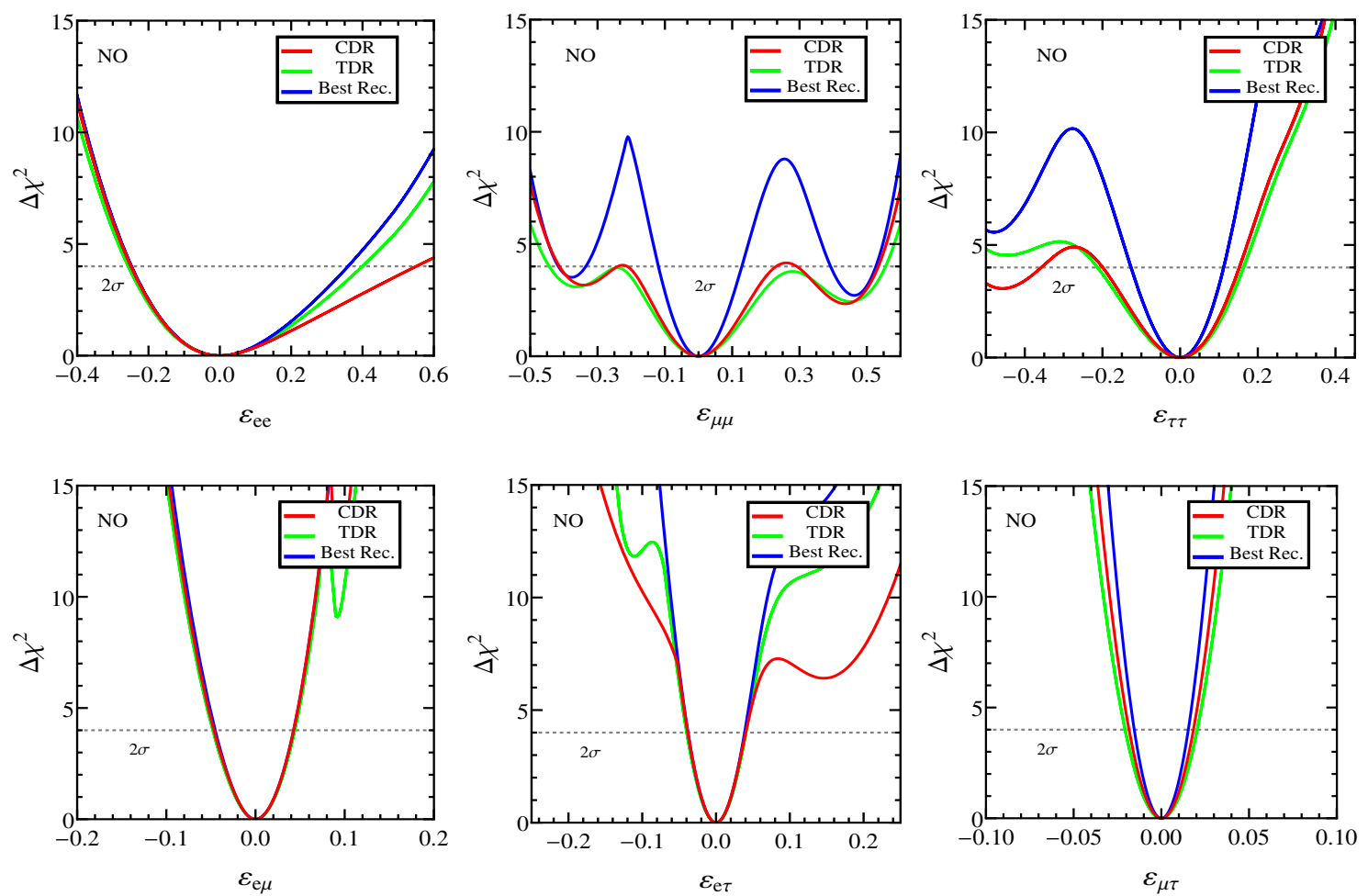

Figure 3. One-dimensional projections of the DUNE sensitivity to the diagonal NSI parameters $\varepsilon_{e e}, \varepsilon_{\mu \mu}, \varepsilon_{\tau \tau}$ in the upper panel and non-diagonal $\varepsilon_{e \mu}, \varepsilon_{e \tau}, \varepsilon_{\mu \tau}$ in the lower panel. The red line represents the result with "CDR" configuration, black line is for the "TDR" configuration, and the blue line corresponds to the "Best Reconstruction" configuration. Normal mass ordering is assumed in the data as well as in the theory. We have considered $\delta_{\mathrm{CP}}($ true $)=-90^{\circ}$ and the non-diagonal NSI parameters as real.

\section{$6 \quad$ Numerical results}

In this section we discuss the main results we have obtained from our numerical simulations regarding the impact of the energy resolution on the sensitivity to NSIs.

Let us start with figure 3, where we have adopted a simplified framework in which all NSI parameters $\varepsilon_{\alpha \beta}$ are taken to be real parameters and we take one at a time when analyzing DUNE's sensitivity. Here, and throughout this paper, we will always assume standard three neutrino oscillations as the true hypothesis and test nonzero NSI against it, that is our $\Delta \chi^{2}=\chi_{\mathrm{NSI}}^{2}-\chi_{\mathrm{SM}}^{2}$. For concreteness we have assumed $\delta_{\mathrm{CP}}($ true $)=-90^{\circ}$, which is close to the current preferred value from global fits [2-4]. We marginalize on the two mixing angles $\theta_{13}$ and $\theta_{23}$, the standard $C P$ phase $\delta_{\mathrm{CP}}$, the mass-squared splitting $\Delta m_{31}^{2}$, and the matter density, as discussed in the previous section. The CDR, TDR and Best Reconstruction scenarios are shown in red, green and blue, respectively. The upper panels show the sensitivity for the diagonal NSI parameters whereas the lower panel shows the sensitivity for the non-diagonal NSI parameters, which are assumed real here.

We can clearly see that, while the CDR and TDR cases yield comparable results, an improved energy reconstruction significantly enhances DUNE's sensitivity to NSIs, in 


\begin{tabular}{|c|c|c|c|}
\hline NSI Parameter & CDR & TDR & Best Rec. \\
\hline$\varepsilon_{e e}$ & {$[-0.249,+0.552]$} & {$[-0.256,+0.399]$} & {$[-0.246,+0.360]$} \\
\hline$\varepsilon_{\mu \mu}$ & $\begin{array}{c}{[-0.415,-0.240],} \\
{[-0.214,0.232],} \\
{[0.289,0.522]}\end{array}$ & {$[-0.445,+0.549]$} & $\begin{array}{c}{[-0.416,-0.335],} \\
{[-0.117,0.128],} \\
{[0.393,0.520]}\end{array}$ \\
\hline$\varepsilon_{\tau \tau}$ & $\begin{array}{c}{[-0.550,-0.357],} \\
{[-0.200,+0.154]}\end{array}$ & {$[-0.214,+0.164]$} & {$[-0.126,+0.112]$} \\
\hline$\varepsilon_{e \mu}$ & {$[-0.046,+0.043]$} & {$[-0.047,+0.045]$} & {$[-0.045,+0.043]$} \\
\hline$\varepsilon_{e \tau}$ & {$[-0.038,+0.041]$} & {$[-0.039,+0.041]$} & {$[-0.033,+0.033]$} \\
\hline$\varepsilon_{\mu \tau}$ & {$[-0.018,+0.018]$} & {$[-0.021,+0.021]$} & {$[-0.015,+0.015]$} \\
\hline
\end{tabular}

Table 1. DUNE sensitivities on the NSI parameters at $2 \sigma$ confidence level (C.L.) with 1 degree of freedom (d.o.f.) (i.e. $\Delta \chi^{2}=4$ ), assuming normal mass ordering and $\delta_{\mathrm{CP}}$ (true) $=-90^{\circ}$ (cf. figure 3). Left, middle, and right columns correspond to CDR, TDR, and Best Reconstruction cases respectively. Here the non-diagonal NSI parameters have been assumed real.

particular for $\varepsilon_{\mu \mu}, \varepsilon_{\tau \tau}$, and $\varepsilon_{e \tau}$, as well as $\varepsilon_{\mu \tau}$ to a certain extent. There is some impact in $\varepsilon_{e e}$, though somewhat small, and almost no effect on $\varepsilon_{e \mu}$. Numbers comparing the allowed regions at $2 \sigma\left(\Delta \chi^{2}=4\right)$ for all NSI parameters and for the three cases can be found in table 1. Note that there are existing constraints from several experiments on all NSI parameters, see e.g. ref. [8].

Until now we have treated the non-diagonal NSI parameters as real parameters. However it might be interesting to see how the bound changes when we treat these parameters as complex. As we discussed before, the NSI $C P$ phases may significantly impact the sensitivity to $\varepsilon_{\mu \tau}$, as the first order contribution of this NSI parameter to the $\nu_{\mu}$ disappearance probability goes like $\left|\varepsilon_{\mu \tau}\right| \cos \phi_{\mu \tau}$, see eq. (2.10).

We show DUNE's sensitivity to complex non-diagonal NSI parameters in figure 4 . While the procedure adopted here is the same as in the previous figure, we have additionally marginalized over the corresponding NSI $C P$ phases. As discussed, the sensitivity to $\left|\varepsilon_{\mu \tau}\right|$ greatly diminishes (right panel), while a smaller but still significant effect can be seen for $\left|\varepsilon_{e \tau}\right|$ (middle panel). $\left|\varepsilon_{e \mu}\right|$ is largely unaffected by the presence of a nonzero phase. Even accounting for the $C P$ phases, the improvement due to a better energy resolution remains for $\varepsilon_{e \tau}$, as we can see in the middle panel of figure 4. Quantitatively, we found the upper bounds on $\left|\varepsilon_{e \tau}\right|$ at $2 \sigma\left(\Delta \chi^{2}=4\right)$ to be $0.12,0.10$ and 0.08 for the CDR, TDR and Best Reconstruction cases, respectively, revealing a possible $20 \%$ improvement between the last two. In table 2 , we quote the $2 \sigma$ sensitivity on the non-diagonal, complex NSI parameters for all three scenarios. We highlight that DUNE will significantly improve 

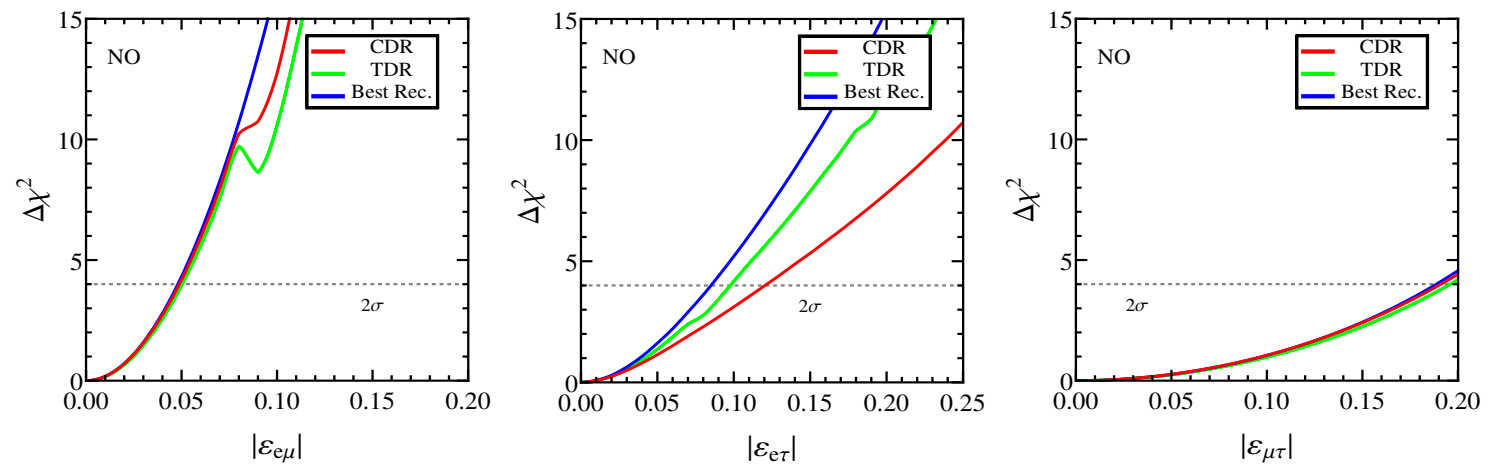

Figure 4. Same as in figure 3 for the non-diagonal NSI parameters $\varepsilon_{e \mu}, \varepsilon_{e \tau}$, and $\varepsilon_{\mu \tau}$, but now assuming them to be complex and marginalizing over their phases.

\begin{tabular}{|c|c|c|c|}
\hline NSI Parameter & CDR & TDR & Best Rec. \\
\hline$\left|\varepsilon_{e \mu}\right|$ & $\leq 0.048$ & $\leq 0.052$ & $\leq 0.047$ \\
\hline$\left|\varepsilon_{e \tau}\right|$ & $\leq 0.123$ & $\leq 0.096$ & $\leq 0.085$ \\
\hline$\left|\varepsilon_{\mu \tau}\right|$ & $\leq 0.191$ & $\leq 0.196$ & $\leq 0.189$ \\
\hline
\end{tabular}

Table 2. Same as in table 1, but for the magnitude of the non-diagonal NSI parameters which are taken to be complex here (cf. figure 4).

current sensitivities to $\varepsilon_{e \mu}$ and $\varepsilon_{e \tau}$ (for both real and complex NSI cases). For the $\varepsilon_{\mu \tau}$, DUNE could compete with the IceCube constraint if the NSI is real. The presence of a nonzero $\phi_{\mu \tau}$ considerably worsens DUNE sensitivity, but not IceCube's [83].

To show how an improved energy resolution can affect the sensitivity to NSIs for different true values of the $C P$ violation phase $\delta_{\mathrm{CP}}$, we present figures 5 and 6 . The simulation details are the same as used before. The contours in each panel is shown for $2 \sigma$ C.L. with 1 d.o.f. (i.e. $\Delta \chi^{2}=4$ ). The CDR, TDR and Best Reconstruction cases are represented by red, green and blue contours respectively. Figure 5 shows the allowed regions for each NSI parameter $\left(\varepsilon_{e e}, \varepsilon_{e \mu}, \varepsilon_{e \tau}, \varepsilon_{\mu \tau}, \varepsilon_{\mu \mu}\right.$, and $\left.\varepsilon_{\tau \tau}\right)$, taking one NSI at a time and assuming all NSI parameters to be real. The null hypothesis corresponds to no NSI and a given value of $\delta_{\mathrm{CP}}$ (true) as indicated on the $y$-axis. Therefore, this figure should be understood as the allowed region of each NSI parameter for a given input value of $\delta_{\mathrm{CP}}$. The variation among $\mathrm{CDR}, \mathrm{TDR}$ and Best Reconstruction shows the role of the energy resolution. If we take $\varepsilon_{e e}$ as an example, we can see that the energy resolution can significantly improve DUNE's sensitivity to this NSI, except for $\delta_{\mathrm{CP}}($ true $) \sim 80^{\circ},-110^{\circ}$. It is evident from this figure that the better energy resolution in DUNE plays a significant role in improving the constraints on most of the NSI parameters. Also note that some spurious degeneracies in the $\varepsilon_{\tau \tau}$ case are only present for the CDR and TDR resolutions, while completely disappear for the Best Reconstruction scenario. 

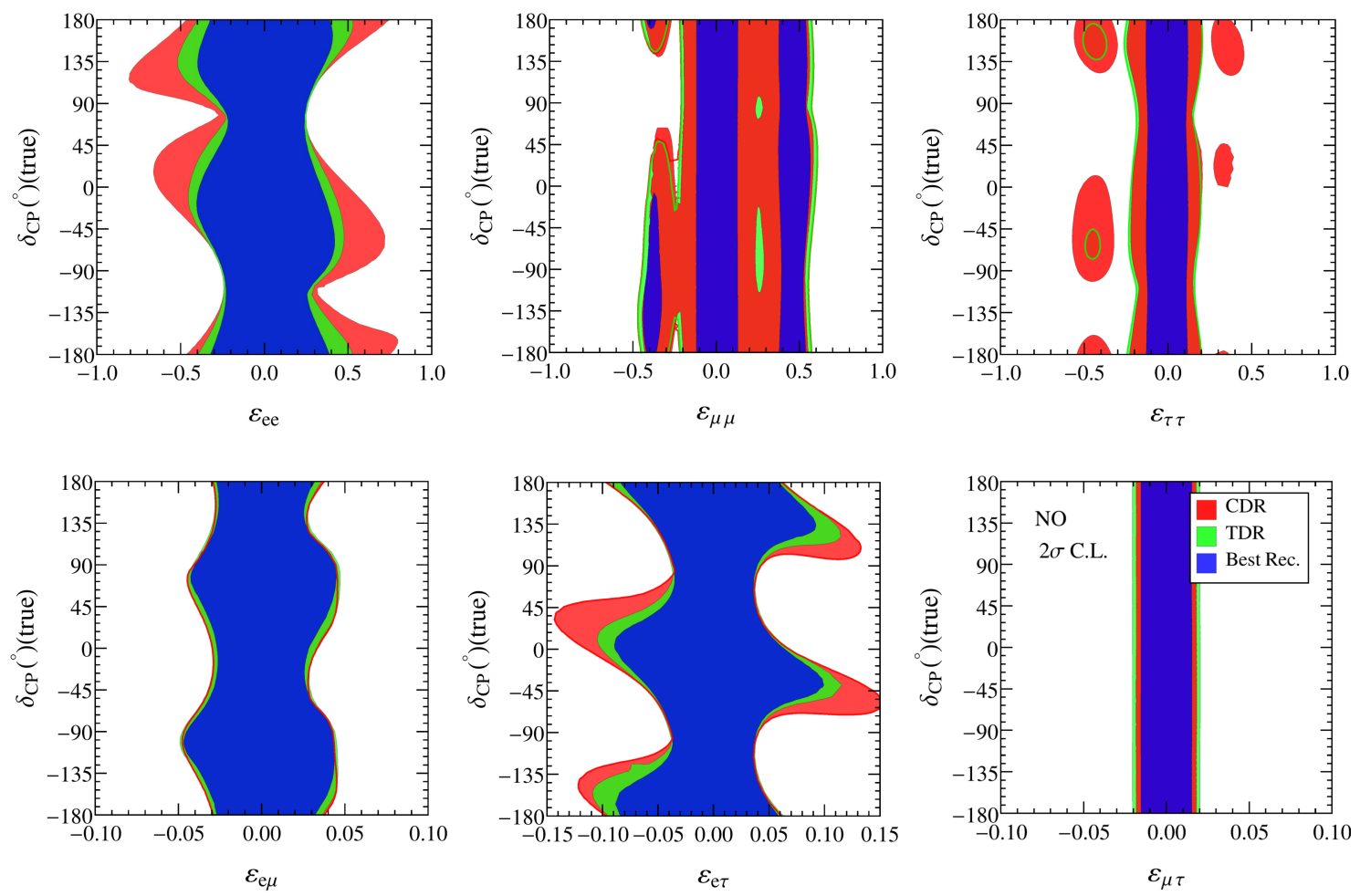

Figure 5. Projected allowed regions in the plane spanned by the $\delta_{\mathrm{CP}}$ (true) and the NSI parameters at $2 \sigma$ C.L. $\left(\Delta \chi^{2}=4\right)$. The red contours represent the DUNE sensitivity for the CDR configuration, green contours correspond to the TDR setup, and the blue contours are for the Best Reconstruction case. Normal mass ordering is assumed to be fixed in the data as well as in theory. We have considered the NSI parameters to be real.
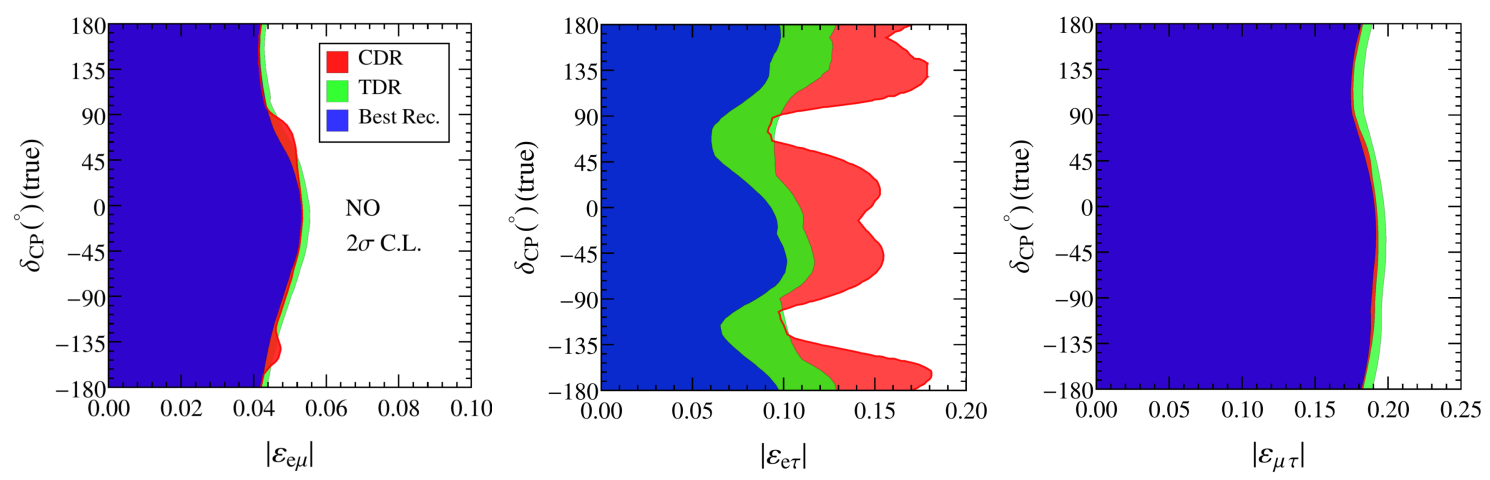

Figure 6. Same as in figure 5 for the non-diagonal NSI parameters, but now assuming them to be complex. 

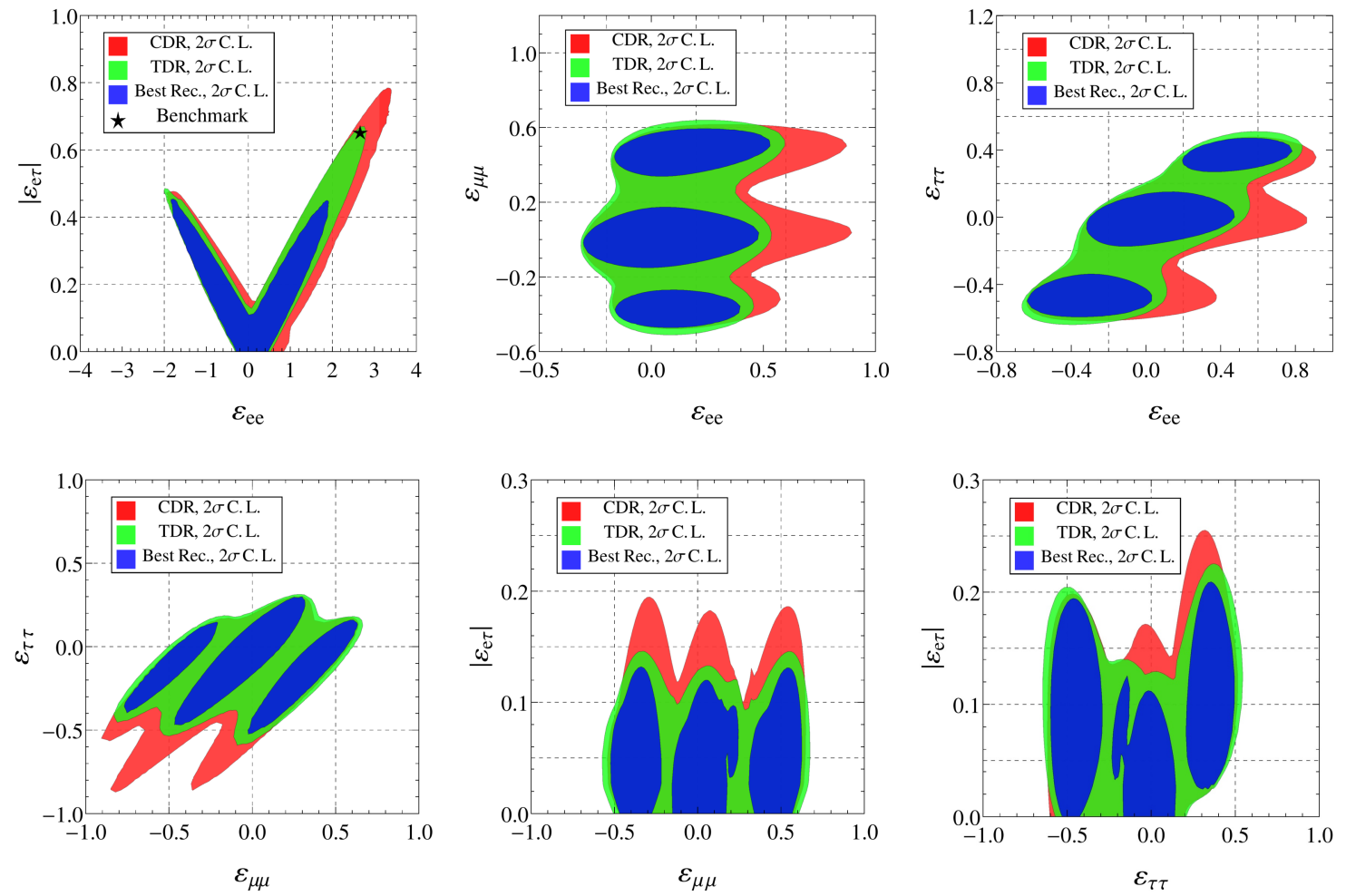

Figure 7. $2 \sigma$ C.L. (2 d.o.f.) sensitivity regions spanned by the different sets of NSI parameters for three different cases CDR, TDR, and Best Reconstruction respectively. We have assumed $\delta_{\mathrm{CP}}($ true $)=-90^{\circ}$ and normal ordering is assumed in both true and test hypotheses of the analysis. The benchmark point marked in the upper left panel will be used later in section 7 .

In the previous discussion we focused on real NSI parameters. Now, we present in figure 6 the allowed region for the absolute value of non-diagonal NSI parameters, marginalizing over their corresponding $C P$ phase, as a function of the true value of $\delta_{\mathrm{CP}}$. All analysis details are the same as before. As expected, the allowed regions are larger when one consider complex NSI parameters, particularly for $\varepsilon_{\mu \tau}$ due to the $\cos \phi_{\mu \tau}$ factor in the leading-order contribution of this NSI to the $\nu_{\mu}$ survival probability, see eq. (2.10). We also observe that the energy resolution plays an important role in the sensitivity to $\varepsilon_{e \tau}$ even in the presence of the NSI $C P$ phase.

Now that we have established the important role of the energy resolution in constraining certain NSI parameters, it is important to assess how robust these conclusions are if we do not assume one NSI parameter at a time. In general, UV complete models will predict a combination of NSI parameters, with possible correlations stemming from the new particles and interactions that generate the NSI at a higher scale [10-16]. Although a comprehensive study of specific models is beyond the scope of this paper, we can redo our analysis taking into account two NSI parameters at a time, instead of individual $\varepsilon_{\alpha \beta}$. Note that further redoing the analysis with more than two NSI parameters will not be very illuminating for our purposes.

In figure 7 we show the allowed regions at $2 \sigma$ C.L. with 2 d.o.f. $\left(\Delta \chi^{2}=6.18\right)$ for different pairs of NSI parameters. We consider $\varepsilon_{e e}, \varepsilon_{\mu \mu}, \varepsilon_{\tau \tau}$, and $\varepsilon_{e \tau}$, as those are the 

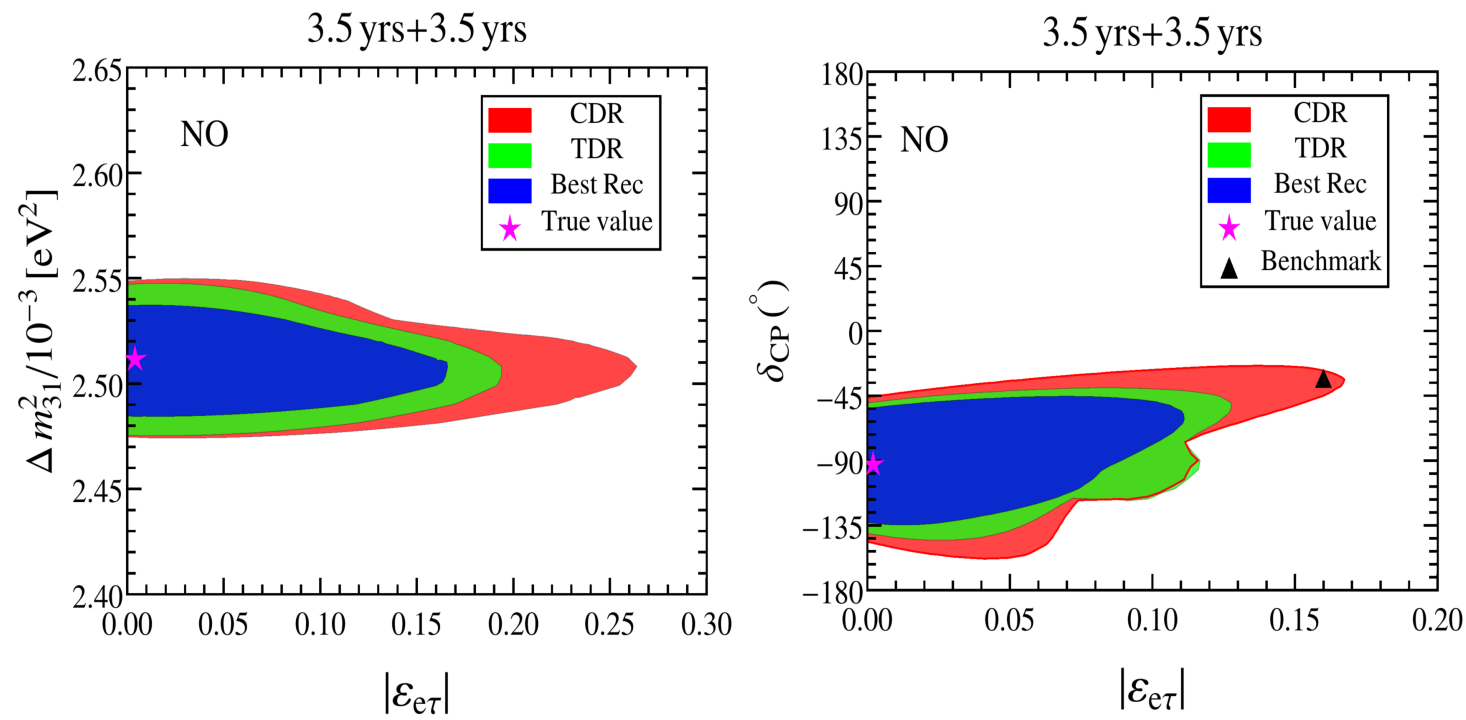

Figure 8. Allowed regions in the $\varepsilon_{e \tau}$ versus $\Delta m_{31}^{2}$ (left) and $\varepsilon_{e \tau}$ versus $\delta_{\mathrm{CP}}$ (right) planes for the DUNE CDR, TDR and Best Reconstruction cases (red, green, and blue contours, respectively) at $2 \sigma$ C.L. $\left(\Delta \chi^{2}=6.18\right)$. The assumed true value is indicated as a magenta star while the black triangle on the right panel is a NSI benchmark, see text.

parameters that profit the most from an improved energy resolution (cf. figures 3 and 4). As before, we indicate the CDR, TDR and Best Reconstruction configurations by the red, green and blue shaded regions, respectively. In order to draw the two-dimensional contours we have marginalized away the two mixing angles $\theta_{13}$ and $\theta_{23}$, the mass splitting $\Delta m_{31}^{2}$, the $C P$ phase $\delta_{\mathrm{CP}}$, and the matter density $\rho$, within the uncertainties discussed in section 4. Moreover the nonstandard $C P$ phases for non-diagonal NSIs have also been marginalized away. We have fixed $\delta_{\mathrm{CP}}\left(\right.$ true) at $-90^{\circ}$ as well as normal ordering for the mass spectrum. We have considered a few combinations of two NSI parameters at a time keeping others fixed to zero. As expected (see e.g. refs. [21, 93]), we observe significant degeneracies between NSI parameters, particularly for the pairs containing $\left(\varepsilon_{e e},\left|\varepsilon_{e \tau}\right|\right)$. We also see here a substantial impact of the improved energy resolution in several of these NSI combinations. In the upper left panel of figure 7 we can see that the allowed region in the Best Reconstruction scenario in the $\left(\varepsilon_{e e},\left|\varepsilon_{e \tau}\right|\right)$ plane for positive $\varepsilon_{e e}$ is reduced to about half of the allowed region in the CDR case. Moreover, the allowed regions in both $\varepsilon_{\mu \mu}$ and $\epsilon_{\tau \tau}$ versus other parameters are connected for the CDR and TDR cases, whereas three disconnected islands appear for the Best Reconstruction case.

\section{Discussion}

In this section we discuss the results obtained previously and the role of the energy resolution in a qualitative manner. First, we present figure 8 , in which we show an illustrative example of DUNE's sensitivity to NSI for CDR, TDR and Best Reconstruction (red, green and blue, resp.) in the planes of $\left(\varepsilon_{e \tau}, \Delta m_{31}^{2}\right)$ (left panel) and $\left(\varepsilon_{e \tau}, \delta_{\mathrm{CP}}\right)$ (right panel) at $2 \sigma$ C.L. for 2 d.o.f. $\left(\Delta \chi^{2}=6.18\right)$. The magenta star in each panel represents the choice of 
true parameters where we have assumed no NSI. Note that we have marginalized away the NSI $C P$ phase $\phi_{e \tau}$ in both panels.

The improvement on the measurement of $\Delta m_{31}^{2}$ due to a better energy resolution is obvious: a more precise determination of the minimum of oscillation allows for a better measurement of the oscillation frequency and therefore of the mass splitting. In fact, the better energy resolution makes the first oscillation minimum in the disappearance channel more prominent and helps resolving the second oscillation minimum, around $0.8 \mathrm{GeV}$. Nevertheless, the right panel is more interesting for us, as we see a degeneracy between $\delta_{\mathrm{CP}}$ and $\varepsilon_{e \tau}$. To understand the role of the energy resolution, let us take the NSI benchmark point denoted by the black triangle in the right panel, which is allowed by the CDR but excluded by the TDR or Best Reconstruction simulations. The relevant parameters for this benchmark point are $\delta_{\mathrm{CP}}=-35^{\circ},\left|\varepsilon_{e \tau}\right|=0.16$, and $\phi_{e \tau}=10^{\circ}$ for the CDR, and $\phi_{e \tau}=15^{\circ}$ for the TDR and Best Reconstruction cases. The other standard oscillation parameters were chosen to be the best fit values in the analysis.

In figure 9 we show the appearance spectra for the neutrino mode (upper left panel) and antineutrino mode (upper right panel) for the CDR, TDR and Best Reconstruction (red, green and blue, respectively), for the assumed true parameters (magenta star in figure 8 right panel, solid lines in figure 9) and NSI benchmark point (black triangle in figure 8 right panel, dashed lines in figure 9). First we can understand why it is hard to disentangle this NSI effect: the spectra themselves look very similar between the standard model assumed true values and the NSI benchmark. This is particularly true for the first maximum. The second maximum shows a larger deviation, but with lower statistics. To better see the differences between the standard and NSI cases, we show in the lower panels the absolute difference on the number of events divided by the statistical uncertainty in each bin, i.e. $\Delta=\left|N_{\mathrm{SM}}-N_{\mathrm{NSI}}\right| / \sqrt{N_{\mathrm{SM}}}$. This statistical figure of merit should be viewed only as a crude approximation for the statistical relevance of each bin, but it serves to demonstrate the relevance of the first and second oscillation maxima in a qualitative level. As we can see, a better energy resolution not only leads to a slight increase in the statistical power around the first oscillation maximum, but also on the second maximum. This draws us to the conclusion that the improved energy resolution enhances the sensitivity not only due to a better reconstruction of the first maximum: the role of the second maximum is indeed very much significant. These considerations apply to both neutrino and antineutrino modes.

To be more explicit, we compare in figure 10 the appearance spectra between the standard case (solid lines) and the NSI case (dashed lines) corresponding to a point in parameter space which is ruled out by the Best Reconstruction but not by the TDR configuration (see the black star in figure 7 upper left panel). Upper left (right) panel in figure 10 presents the $\nu_{\mu} \rightarrow \nu_{e}\left(\bar{\nu}_{\mu} \rightarrow \bar{\nu}_{e}\right)$ appearance event spectra as a function of reconstructed energy. Green and blue lines correspond to the TDR and Best Reconstruction configurations respectively. Solid lines represent the standard three-neutrino spectra with $\delta_{\mathrm{CP}}=-90^{\circ}$ and dashed lines represent the spectra with NSI in the $e-e$ and $e-\tau$ sector simultaneously. In case of NSI, we have taken the best fit parameters corresponding to the black star in the upper left panel of figure 7, i.e., $\delta_{\mathrm{CP}}=-22^{\circ}, \varepsilon_{e e}=2.70,\left|\varepsilon_{e \tau}\right|=0.65$, and $\phi_{e \tau}=-177^{\circ}$ for the TDR, and $\delta_{\mathrm{CP}}=-23^{\circ}, \varepsilon_{e e}=2.70,\left|\varepsilon_{e \tau}\right|=0.65$, and $\phi_{e \tau}=-170^{\circ}$ for the Best 


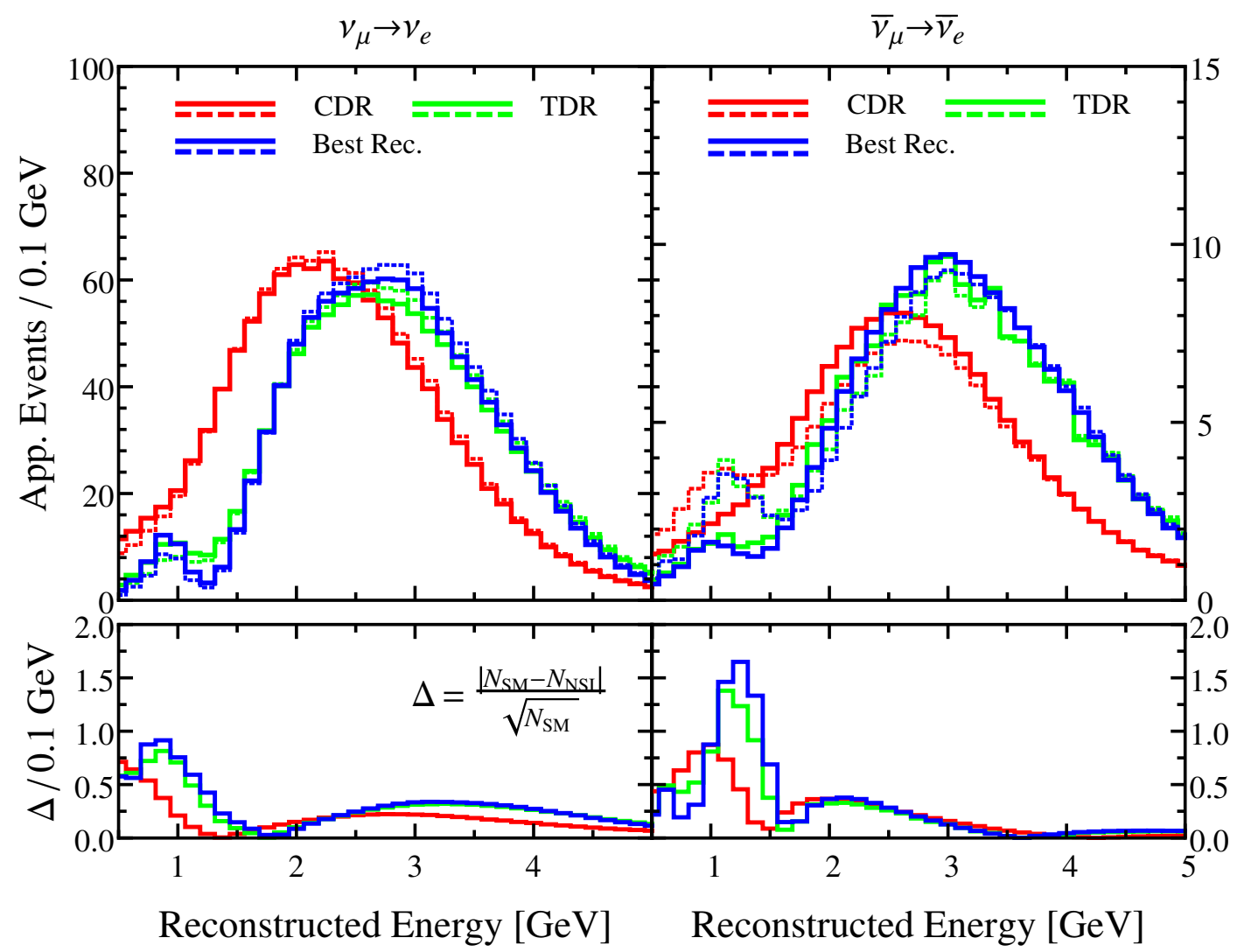

Figure 9. Upper left (right) panel presents the $\nu_{\mu} \rightarrow \nu_{e}\left(\bar{\nu}_{\mu} \rightarrow \bar{\nu}_{e}\right)$ appearance event spectra as a function of reconstructed energy. Red, green and blue lines correspond to the CDR, TDR, and Best Reconstruction configurations, respectively. Solid lines represent the standard three-neutrino spectra with $\delta_{\mathrm{CP}}=-90^{\circ}$ and dashed lines represent the spectra with NSI in the $e-\tau$ sector. In case of NSI, we have taken the best fit parameters corresponding to the black triangle in figure 8 , i.e., $\delta_{\mathrm{CP}}=-35^{\circ},\left|\varepsilon_{e \tau}\right|=0.16$, and $\phi_{e \tau}=10^{\circ}$ for the CDR, and $\phi_{e \tau}=15^{\circ}$ for the TDR and Best Reconstruction cases. The best fit values of $\theta_{13}, \theta_{23}$, and $\Delta m_{31}^{2}$ have also been chosen from the simulation. Lower left (right) panel represents the absolute difference in the upper left (right) appearance events between the standard and the NSI scenarios divided by the statistical uncertainty in each bin. The color coding is the same as in the upper panels.

Reconstruction case respectively. The best fit values of $\theta_{13}, \theta_{23}$, and $\Delta m_{31}^{2}$ have also been chosen from the simulation for both the standard and the NSI scenarios respectively. In the lower panels we represent the absolute difference $\Delta$ in the appearance events between the standard and the NSI scenarios divided by the statistical uncertainty in each bin. The color coding is the same as in the upper panels. Here, the effect of the improved energy resolution is very clear: both first and second maxima become statistically more powerful in rejecting this NSI hypothesis. Although the second maximum has less events and less bins, the relative change in the number of events is significantly larger than in the first maximum. This evidences that both oscillation maxima can contribute comparably to a better sensitivity, if they are resolved more precisely. 


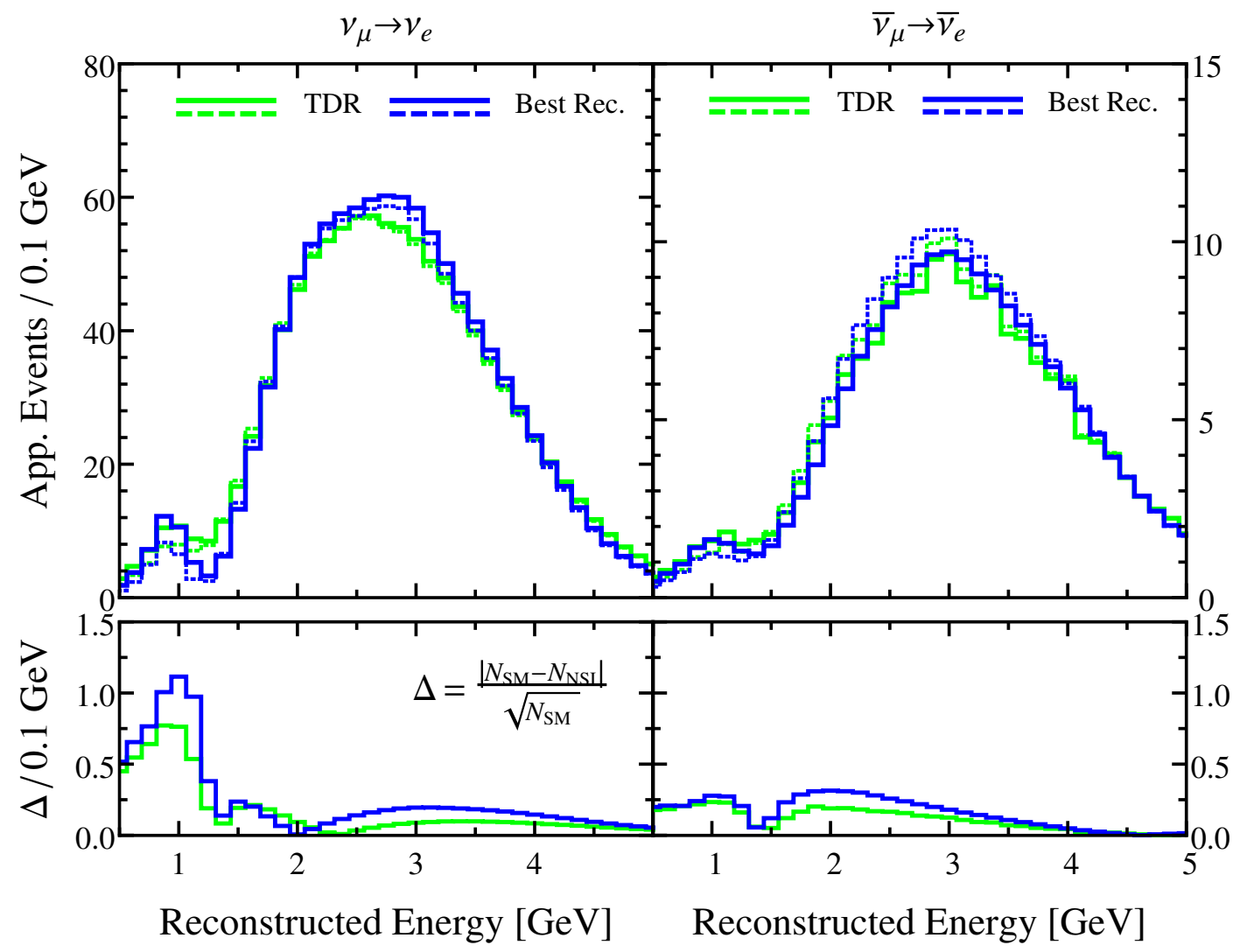

Figure 10. Upper left (right) panel presents the $\nu_{\mu} \rightarrow \nu_{e}\left(\bar{\nu}_{\mu} \rightarrow \bar{\nu}_{e}\right)$ appearance event spectra as a function of reconstructed energy. Green and blue lines correspond to the TDR and Best Reconstruction configurations respectively. Solid lines represent the standard three-neutrino spectra with $\delta_{\mathrm{CP}}=-90^{\circ}$ and dashed lines represent the spectra with NSIs in the $e-e$ and $e-\tau$ sectors simultaneously. In case of NSI, we have taken the best fit parameters corresponding to the black star in the upper left panel of figure 7, i.e., $\delta_{\mathrm{CP}}=-22^{\circ}, \varepsilon_{e e}=2.70,\left|\varepsilon_{e \tau}\right|=0.65$, and $\phi_{e \tau}=-177^{\circ}$ for the TDR, and $\delta_{\mathrm{CP}}=-23^{\circ}, \varepsilon_{e e}=2.70,\left|\varepsilon_{e \tau}\right|=0.65$, and $\phi_{e \tau}=-170^{\circ}$ for the Best Reconstruction case. The best fit values of $\theta_{13}, \theta_{23}$, and $\Delta m_{31}^{2}$ have also been chosen from the simulation for both the standard and the NSI scenarios. Lower left (right) panel represents the absolute difference in the upper left (right) appearance events between the standard and the NSI scenarios divided by the statistical uncertainty in each bin. The color coding is the same as in the upper panels.

\section{Conclusions}

In this work we have investigated the impact of improved energy resolution on DUNE sensitivity to new physics. As an illustrative example, we have shown how a better energy resolution could lead to improved constraints on neutrino non-standard interactions with matter. We have found that a better energy resolution would help to disentangle degeneracies among effects arising from standard three-neutrino oscillations, such as the usual $C P$ violation, and those stemming from NSIs. In fact, by studying in more detail certain benchmark points, we have identified that the improved sensitivity to NSIs comes not only 
from the first oscillation peak, but the second maximum also contributes significantly to the experimental sensitivity as long as it can be appropriately resolved. Therefore, a better neutrino energy determination allows the experiment to further leverage its broad-band beam in BSM physics searches.

More specifically, we have found that potentially significant sensitivity improvements could be achieved for $\varepsilon_{e e}, \varepsilon_{\mu \mu}, \varepsilon_{\tau \tau}$, and $\varepsilon_{e \tau}$, particularly when the NSI $C P$ violating phase is considered for $\varepsilon_{e \tau}$. We have also found that certain degeneracies could be better resolved with an improved energy resolution. For any combination of two of the aforementioned NSI parameters, we find a significant reduction in the allowed regions, when going from the CDR case to the TDR and especially to the Best Reconstruction scenario (see figure 7). Finally, as a by-product of our analysis, we have also shown how the $C P$ violation sensitivity and the $C P$ phase precision would improve with the neutrino energy resolution (see figure 2). We hope our analysis will further motivate the pursuit of an enhanced neutrino energy reconstruction in liquid argon time projection chambers.

\section{Acknowledgments}

We would like to thank Shirley Li for useful discussions on the DUNE energy resolution, Chris Marshall and Elizabeth Worcester for discussions and clarifications regarding the GLoBES simulation for DUNE TDR, and Sanjib Kumar Agarwalla for some earlier discussions on NSI at DUNE. Fermilab is operated by the Fermi Research Alliance, LLC under contract No. DE-AC02-07CH11359 with the United States Department of Energy. This project has received support from the European Union's Horizon 2020 research and innovation programme under the Marie Skłodowska-Curie grant agreement No 860881-HIDDeN. The work of BD is supported in part by the US Department of Energy under Grant No. DE-SC0017987, by the Neutrino Theory Network Program, and by a Fermilab Intensity Frontier Fellowship. BD and SSC would like to thank the Fermilab Theory Group for local hospitality during a summer visit in 2019, where this project was initiated.

Open Access. This article is distributed under the terms of the Creative Commons Attribution License (CC-BY 4.0), which permits any use, distribution and reproduction in any medium, provided the original author(s) and source are credited.

\section{References}

[1] Particle Data Group collaboration, Review of Particle Physics, PTEP 2020 (2020) $083 \mathrm{C} 01$ [INSPIRE].

[2] F. Capozzi, E. Lisi, A. Marrone and A. Palazzo, Current unknowns in the three neutrino framework, Prog. Part. Nucl. Phys. 102 (2018) 48 [arXiv:1804.09678] [InSPIRE].

[3] P.F. de Salas et al., 2020 global reassessment of the neutrino oscillation picture, JHEP 02 (2021) 071 [arXiv : 2006.11237] [inSPIRE].

[4] I. Esteban, M.C. Gonzalez-Garcia, M. Maltoni, T. Schwetz and A. Zhou, The fate of hints: updated global analysis of three-flavor neutrino oscillations, JHEP 09 (2020) 178 [arXiv:2007.14792] [INSPIRE]. 
[5] L. Wolfenstein, Neutrino Oscillations in Matter, Phys. Rev. D 17 (1978) 2369 [inSPIRE].

[6] T. Ohlsson, Status of non-standard neutrino interactions, Rept. Prog. Phys. 76 (2013) 044201 [arXiv: 1209.2710] [INSPIRE].

[7] O.G. Miranda and H. Nunokawa, Non standard neutrino interactions: current status and future prospects, New J. Phys. 17 (2015) 095002 [arXiv: 1505. 06254] [INSPIRE].

[8] Y. Farzan and M. Tortola, Neutrino oscillations and Non-Standard Interactions, Front. in Phys. 6 (2018) 10 [arXiv:1710.09360] [INSPIRE].

[9] P.S.B. Dev et al., Neutrino Non-Standard Interactions: A Status Report, Sci. Post. Phys. Proc. 2 (2019) 001 [arXiv: 1907.00991] [INSPIRE].

[10] Y. Farzan, A model for large non-standard interactions of neutrinos leading to the LMA-Dark solution, Phys. Lett. B 748 (2015) 311 [arXiv:1505.06906] [INSPIRE].

[11] Y. Farzan and I.M. Shoemaker, Lepton Flavor Violating Non-Standard Interactions via Light Mediators, JHEP 07 (2016) 033 [arXiv:1512.09147] [INSPIRE].

[12] Y. Farzan and J. Heeck, Neutrinophilic nonstandard interactions, Phys. Rev. D 94 (2016) 053010 [arXiv: 1607.07616$]$ [INSPIRE].

[13] D.V. Forero and W.-C. Huang, Sizable NSI from the $\mathrm{SU}(2)_{L}$ scalar doublet-singlet mixing and the implications in DUNE, JHEP 03 (2017) 018 [arXiv:1608.04719] [INSPIRE].

[14] K.S. Babu, A. Friedland, P.A.N. Machado and I. Mocioiu, Flavor Gauge Models Below the Fermi Scale, JHEP 12 (2017) 096 [arXiv: 1705. 01822] [INSPIRE].

[15] U.K. Dey, N. Nath and S. Sadhukhan, Non-Standard Neutrino Interactions in a Modified

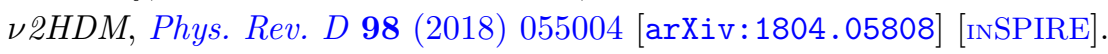

[16] K.S. Babu, P.S.B. Dev, S. Jana and A. Thapa, Non-Standard Interactions in Radiative Neutrino Mass Models, JHEP 03 (2020) 006 [arXiv:1907.09498] [INSPIRE].

[17] I. Esteban, M.C. Gonzalez-Garcia and M. Maltoni, On the Determination of Leptonic CP-violation and Neutrino Mass Ordering in Presence of Non-Standard Interactions: Present Status, JHEP 06 (2019) 055 [arXiv: 1905.05203] [INSPIRE].

[18] P. Coloma, I. Esteban, M.C. Gonzalez-Garcia and M. Maltoni, Improved global fit to Non-Standard neutrino Interactions using COHERENT energy and timing data, JHEP 02 (2020) 023 [Addendum ibid. 12 (2020) 071] [arXiv: 1911.09109] [INSPIRE].

[19] O.G. Miranda, M.A. Tortola and J.W.F. Valle, Are solar neutrino oscillations robust?, JHEP 10 (2006) 008 [hep-ph/0406280] [inSPIRE].

[20] I. Girardi, D. Meloni and S.T. Petcov, The Daya Bay and T2K results on $\sin ^{2} 2 \theta_{13}$ and Non-Standard Neutrino Interactions, Nucl. Phys. B 886 (2014) 31 [arXiv:1405.0416] [INSPIRE].

[21] J. Liao, D. Marfatia and K. Whisnant, Degeneracies in long-baseline neutrino experiments from nonstandard interactions, Phys. Rev. D 93 (2016) 093016 [arXiv:1601.00927] [INSPIRE].

[22] M. Masud and P. Mehta, Nonstandard interactions spoiling the CP-violation sensitivity at DUNE and other long baseline experiments, Phys. Rev. D 94 (2016) 013014 [arXiv: 1603.01380] [INSPIRE].

[23] A. de Gouvêa and K.J. Kelly, False Signals of CP-Invariance Violation at DUNE, arXiv: 1605.09376 [INSPIRE]. 
[24] M. Masud and P. Mehta, Nonstandard interactions and resolving the ordering of neutrino masses at DUNE and other long baseline experiments, Phys. Rev. D 94 (2016) 053007 [arXiv: 1606.05662] [INSPIRE].

[25] S.K. Agarwalla, S.S. Chatterjee and A. Palazzo, Degeneracy between $\theta_{23}$ octant and neutrino non-standard interactions at DUNE, Phys. Lett. B 762 (2016) 64 [arXiv:1607.01745] [INSPIRE].

[26] K.N. Deepthi, S. Goswami and N. Nath, Can nonstandard interactions jeopardize the hierarchy sensitivity of DUNE?, Phys. Rev. D 96 (2017) 075023 [arXiv:1612.00784] [INSPIRE].

[27] K.N. Deepthi, S. Goswami and N. Nath, Challenges posed by non-standard neutrino interactions in the determination of $\delta_{C P}$ at DUNE, Nucl. Phys. B 936 (2018) 91 [arXiv: 1711.04840] [INSPIRE].

[28] L.J. Flores, E.A. Garcés and O.G. Miranda, Exploring NSI degeneracies in long-baseline experiments, Phys. Rev. D 98 (2018) 035030 [arXiv: 1806.07951] [InSPIRE].

[29] J.M. Hyde, Biprobability approach to CP phase degeneracy from non-standard neutrino interactions, Nucl. Phys. B 949 (2019) 114804 [arXiv:1806.09221] [INSPIRE].

[30] M. Masud, S. Roy and P. Mehta, Correlations and degeneracies among the NSI parameters with tunable beams at DUNE, Phys. Rev. D 99 (2019) 115032 [arXiv:1812.10290] [INSPIRE].

[31] F. Capozzi, S.S. Chatterjee and A. Palazzo, Neutrino Mass Ordering Obscured by Nonstandard Interactions, Phys. Rev. Lett. 124 (2020) 111801 [arXiv:1908.06992] [INSPIRE].

[32] O. Yasuda, Neutrino Oscillations at low energy long baseline experiments in the presence of nonstandard interactions and parameter degeneracy, PTEP 2020 (2020) 063B03 [arXiv: 2002.01616] [INSPIRE].

[33] I. Esteban, M.C. Gonzalez-Garcia and M. Maltoni, On the effect of NSI in the present determination of the mass ordering, arXiv:2004.04745 [INSPIRE].

[34] S. Kumar Agarwalla, S. Das, M. Masud and P. Swain, Evolution of Neutrino Mass-Mixing Parameters in Matter with Non-Standard Interactions, arXiv:2103.13431 [INSPIRE].

[35] T. Ota, J. Sato and N.-a. Yamashita, Oscillation enhanced search for new interaction with neutrinos, Phys. Rev. D 65 (2002) 093015 [hep-ph/0112329] [INSPIRE].

[36] M. Blennow, T. Ohlsson and J. Skrotzki, Effects of non-standard interactions in the MINOS experiment, Phys. Lett. B 6660 (2008) 522 [hep-ph/0702059] [INSPIRE].

[37] J. Kopp, M. Lindner, T. Ota and J. Sato, Non-standard neutrino interactions in reactor and superbeam experiments, Phys. Rev. D 77 (2008) 013007 [arXiv: 0708.0152] [inSPIRE].

[38] M. Blennow, D. Meloni, T. Ohlsson, F. Terranova and M. Westerberg, Non-standard interactions using the OPERA experiment, Eur. Phys. J. C 56 (2008) 529 [arXiv:0804.2744] [INSPIRE].

[39] J. Kopp, P.A.N. Machado and S.J. Parke, Interpretation of MINOS Data in Terms of Non-Standard Neutrino Interactions, Phys. Rev. D 82 (2010) 113002 [arXiv:1009.0014] [INSPIRE].

[40] R. Adhikari, S. Chakraborty, A. Dasgupta and S. Roy, Non-standard interaction in neutrino oscillations and recent Daya Bay, T2K experiments, Phys. Rev. D 86 (2012) 073010 [arXiv: 1201.3047] [INSPIRE]. 
[41] A. Friedland and I.M. Shoemaker, Searching for Novel Neutrino Interactions at NOvA and Beyond in Light of Large $\theta_{13}$, arXiv:1207.6642 [INSPIRE].

[42] J.A.B. Coelho, T. Kafka, W.A. Mann, J. Schneps and O. Altinok, Constraints for non-standard interaction $\epsilon_{e \tau} V_{e}$ from $\nu_{e}$ appearance in MINOS and T2K, Phys. Rev. D 86 (2012) 113015 [arXiv:1209.3757] [INSPIRE].

[43] Z. Rahman, A. Dasgupta and R. Adhikari, The Discovery reach of CP violation in neutrino oscillation with non-standard interaction effects, J. Phys. G 42 (2015) 065001 [arXiv: 1503.03248] [INSPIRE].

[44] A. de Gouvêa and K.J. Kelly, Non-standard Neutrino Interactions at DUNE, Nucl. Phys. B 908 (2016) 318 [arXiv:1511.05562] [INSPIRE].

[45] P. Coloma, Non-Standard Interactions in propagation at the Deep Underground Neutrino Experiment, JHEP 03 (2016) 016 [arXiv:1511.06357] [INSPIRE].

[46] D.V. Forero and P. Huber, Hints for leptonic CP-violation or New Physics?, Phys. Rev. Lett. 117 (2016) 031801 [arXiv:1601.03736] [INSPIRE].

[47] K. Huitu, T.J. Kärkkäinen, J. Maalampi and S. Vihonen, Constraining the nonstandard interaction parameters in long baseline neutrino experiments, Phys. Rev. D 93 (2016) 053016 [arXiv: 1601.07730] [INSPIRE].

[48] P. Bakhti and Y. Farzan, CP-Violation and Non-Standard Interactions at the MOMENT, JHEP 07 (2016) 109 [arXiv:1602.07099] [INSPIRE].

[49] S. C and R. Mohanta, Implications of lepton flavor violation on long baseline neutrino oscillation experiments, Phys. Rev. D 94 (2016) 053008 [arXiv: 1603.02184] [INSPIRE].

[50] M. Blennow, S. Choubey, T. Ohlsson, D. Pramanik and S.K. Raut, A combined study of source, detector and matter non-standard neutrino interactions at DUNE, JHEP 08 (2016) 090 [arXiv: 1606.08851] [inSPIRE].

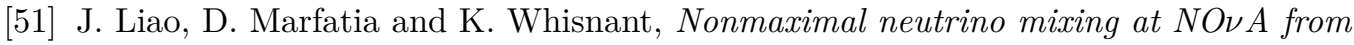
nonstandard interactions, Phys. Lett. B 767 (2017) 350 [arXiv: 1609. 01786] [INSPIRE].

[52] S. Fukasawa, M. Ghosh and O. Yasuda, Sensitivity of the T2HKK experiment to nonstandard interactions, Phys. Rev. D 95 (2017) 055005 [arXiv: 1611.06141] [INSPIRE].

[53] J. Liao, D. Marfatia and K. Whisnant, Nonstandard neutrino interactions at DUNE, T2HK and T2HKK, JHEP 01 (2017) 071 [arXiv:1612.01443] [inSPIRE].

[54] A. Chatterjee, F. Kamiya, C.A. Moura and J. Yu, Impact of Matter Density Profile Shape on Non-Standard Interactions at DUNE, arXiv:1809.09313 [INSPIRE].

[55] T. Han, J. Liao, H. Liu and D. Marfatia, Nonstandard neutrino interactions at COHERENT, DUNE, T2HK and LHC, JHEP 11 (2019) 028 [arXiv:1910.03272] [INSPIRE].

[56] P.B. Denton, J. Gehrlein and R. Pestes, CP -Violating Neutrino Nonstandard Interactions in Long-Baseline-Accelerator Data, Phys. Rev. Lett. 126 (2021) 051801 [arXiv:2008.01110] [INSPIRE].

[57] S.S. Chatterjee and A. Palazzo, Nonstandard Neutrino Interactions as a Solution to the

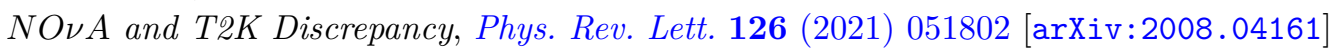
[INSPIRE].

[58] P. Bakhti and M. Rajaee, Sensitivities of future reactor and long-baseline neutrino experiments to NSI, Phys. Rev. D 103 (2021) 075003 [arXiv:2010.12849] [INSPIRE]. 
[59] A. Giarnetti and D. Meloni, New Sources of Leptonic CP-violation at the DUNE Neutrino Experiment, Universe 7 (2021) 240 [arXiv:2106.00030] [INSPIRE].

[60] A. Himmel, New Oscillation Results from the NOvA Experiment, talk presented at Neutrino 2020, Virtual Meeting, 22 June - 2 July 2020, https://doi.org/10.5281/zenodo.4142045.

[61] P. Dunne, Latest Neutrino Oscillation Results from T2, talk presented at Neutrino 2020, Virtual Meeting, 22 June - 2 July 2020, https://doi.org/10.5281/zenodo.3959558.

[62] DUNE collaboration, Long-Baseline Neutrino Facility (LBNF) and Deep Underground Neutrino Experiment (DUNE): Conceptual Design Report, Volume 2: The Physics Program for DUNE at LBNF, arXiv: 1512.06148 [INSPIRE].

[63] DUNE collaboration, Deep Underground Neutrino Experiment (DUNE), Far Detector Technical Design Report, Volume II: DUNE Physics, arXiv:2002.03005 [INSPIRE].

[64] DUNE collaboration, Prospects for beyond the Standard Model physics searches at the Deep Underground Neutrino Experiment, Eur. Phys. J. C 81 (2021) 322 [arXiv:2008.12769] [INSPIRE].

[65] Argoneut collaboration, Demonstration of MeV-Scale Physics in Liquid Argon Time Projection Chambers Using ArgoNeuT, Phys. Rev. D 99 (2019) 012002 [arXiv:1810.06502] [INSPIRE].

[66] W. Castiglioni, W. Foreman, I. Lepetic, B.R. Littlejohn, M. Malaker and A. Mastbaum, Benefits of $\mathrm{MeV}$-scale reconstruction capabilities in large liquid argon time projection chambers, Phys. Rev. D 102 (2020) 092010 [arXiv:2006.14675] [INSPIRE].

[67] K.J. Kelly, P.A. Machado, I. Martinez Soler, S.J. Parke and Y.F. Perez Gonzalez, Sub-GeV Atmospheric Neutrinos and CP-Violation in DUNE, Phys. Rev. Lett. 123 (2019) 081801 [arXiv: 1904.02751] [INSPIRE].

[68] J. Conrad, A. de Gouvêa, S. Shalgar and J. Spitz, Atmospheric Tau Neutrinos in a Multi-kiloton Liquid Argon Detector, Phys. Rev. D 82 (2010) 093012 [arXiv:1008.2984] [INSPIRE].

[69] A. De Gouvêa, K.J. Kelly, G.V. Stenico and P. Pasquini, Physics with Beam Tau-Neutrino Appearance at DUNE, Phys. Rev. D 100 (2019) 016004 [arXiv:1904.07265] [InSPIRE].

[70] P. Machado, H. Schulz and J. Turner, Tau neutrinos at DUNE: New strategies, new opportunities, Phys. Rev. D 102 (2020) 053010 [arXiv: 2007.00015] [InSPIRE].

[71] V. De Romeri, E. Fernandez-Martinez and M. Sorel, Neutrino oscillations at DUNE with improved energy reconstruction, JHEP 09 (2016) 030 [arXiv:1607.00293] [INSPIRE].

[72] A. Friedland and S.W. Li, Understanding the energy resolution of liquid argon neutrino detectors, Phys. Rev. D 99 (2019) 036009 [arXiv:1811.06159] [InSPIRE].

[73] DUNE collaboration, Long-baseline neutrino oscillation physics potential of the DUNE experiment, Eur. Phys. J. C 80 (2020) 978 [arXiv: 2006.16043] [INSPIRE].

[74] J. Rout, S. Shafaq, M. Bishai and P. Mehta, Physics prospects with the second oscillation maximum at the Deep Underground Neutrino Experiment, Phys. Rev. D 103 (2021) 116003 [arXiv:2012.08269] [INSPIRE].

[75] P. Huber and J. Kopp, Two experiments for the price of one? - The role of the second oscillation maximum in long baseline neutrino experiments, JHEP 03 (2011) 013 [Erratum ibid. 05 (2011) 024] [arXiv: 1010.3706] [INSPIRE]. 
[76] Y. Grossman, Nonstandard neutrino interactions and neutrino oscillation experiments, Phys. Lett. B 359 (1995) 141 [hep-ph/9507344] [INSPIRE].

[77] C. Biggio, M. Blennow and E. Fernandez-Martinez, General bounds on non-standard neutrino interactions, JHEP 08 (2009) 090 [arXiv: 0907.0097] [INSPIRE].

[78] A. Datta, J. Liao and D. Marfatia, A light $Z^{\prime}$ for the $R_{K}$ puzzle and nonstandard neutrino interactions, Phys. Lett. B $\mathbf{7 6 8}$ (2017) 265 [arXiv: 1702.01099] [INSPIRE].

[79] T. Kikuchi, H. Minakata and S. Uchinami, Perturbation Theory of Neutrino Oscillation with Nonstandard Neutrino Interactions, JHEP 03 (2009) 114 [arXiv: 0809.3312] [INSPIRE].

[80] J. Arafune, M. Koike and J. Sato, CP violation and matter effect in long baseline neutrino oscillation experiments, Phys. Rev. D 56 (1997) 3093 [Erratum ibid. 60 (1999) 119905] [hep-ph/9703351] [INSPIRE].

[81] M. Freund, Analytic approximations for three neutrino oscillation parameters and probabilities in matter, Phys. Rev. D 64 (2001) 053003 [hep-ph/0103300] [INSPIRE].

[82] E.K. Akhmedov, R. Johansson, M. Lindner, T. Ohlsson and T. Schwetz, Series expansions for three flavor neutrino oscillation probabilities in matter, JHEP 04 (2004) 078 [hep-ph/0402175] [INSPIRE].

[83] ICECUBE collaboration, All-flavor constraints on nonstandard neutrino interactions and generalized matter potential with three years of IceCube DeepCore data, arXiv:2106.07755 [INSPIRE].

[84] DUNE collaboration, Experiment Simulation Configurations Used in DUNE CDR, arXiv: 1606.09550 [INSPIRE].

[85] DUNE collaboration, Experiment Simulation Configurations Approximating DUNE TDR, arXiv: 2103.04797 [INSPIRE].

[86] P. Huber, M. Lindner and W. Winter, Simulation of long-baseline neutrino oscillation experiments with GLoBES (General Long Baseline Experiment Simulator), Comput. Phys. Commun. 167 (2005) 195 [hep-ph/0407333] [INSPIRE].

[87] P. Huber, J. Kopp, M. Lindner, M. Rolinec and W. Winter, New features in the simulation of neutrino oscillation experiments with GLoBES 3.0: General Long Baseline Experiment Simulator, Comput. Phys. Commun. 177 (2007) 432 [hep-ph/0701187] [INSPIRE].

[88] J. Kopp, Sterile neutrinos and non-standard neutrino interactions in GLoBES, (2019) https://www.mpi-hd.mpg.de/personalhomes/globes/tools/snu-1.0.pdf.

[89] F.D. Stacey, Physics of the earth, vol. 2nd, Wiley ed. (1977) [DOI].

[90] A.M. Dziewonski and D.L. Anderson, Preliminary reference earth model, Physics of the Earth and Planetary Interiors 25 (1981) 297.

[91] DAYA BAY collaboration, Measurement of the Electron Antineutrino Oscillation with 1958 Days of Operation at Daya Bay, Phys. Rev. Lett. 121 (2018) 241805 [arXiv:1809.02261] [INSPIRE].

[92] RENO collaboration, Measurement of Reactor Antineutrino Oscillation Amplitude and Frequency at RENO, Phys. Rev. Lett. 121 (2018) 201801 [arXiv:1806.00248] [INSPIRE].

[93] P. Coloma and T. Schwetz, Generalized mass ordering degeneracy in neutrino oscillation experiments, Phys. Rev. D 94 (2016) 055005 [Erratum ibid. 95 (2017) 079903] [arXiv: 1604.05772] [INSPIRE]. 